\title{
SIRCOVA-OFTARED-RIG Joined Congress Abstracts Valencia (Spain), November 7-8, 2014
}

The abstracts are available free online at www.karger.com/doi/10.1159/000369084

\begin{abstract}
About SIRCOVA
SIRCOVA, the Society for Research in Retina was first established in 2009 as a result of collaborative efforts by professionals interested in ophthalmology and visual sciences research that all together built this new society. SIRCOVA was born from a multi-disciplinary viewpoint, with the challenge of encouraging investigation, innovation and the development of biotechnology in retina and visual sciences. SIRCOVA is a non-profit organisation that is in keeping with the member fees and donations. The objectives of the society are: 1) to promote knowledge concerning the retina and the visual sciences based on the highest standards of research in our countries, 2) to promote the exchange of information, education and training in all aspects of retina and visual sciences investigation, 3) to nurture and evolve the links between the ophthalmologists and ophthalmic researchers, and 4) to advise other scientific societies, professionals, patients or anybody interested in advancing knowledge in eye care through outstanding ophthalmic research, innovation and biotechnology.

Since 2011, colleagues engaged in these matters from Italy and Portugal joined SIRCOVA. The Society is governed by an executive board composed from the foundational members, which is representative of the professionals noted above and drawn from the three nations. While several societies exist in our countries which represent the interests of individual ophthalmic modalities, SIRCOVA specifically represents retina and visual sciences research and biotechnology as a whole. We welcome membership from medical and non medical specialists, scientists and students who are interested in retina and visual sciences.

The Society meets each eighteen months either in the spring or autumn of the corresponding year. This year the Spanish Portuguese Glaucoma Workshop has joined our Congress. The 2014 SIRCOVA and RIG joined Congress has been officially recognized as an activity of the Ocular Pathology National Net (OFTARED) of the Institute of Health Carlos III (Spanish Government).

For further information please visit the SIRCOVA site at www.sircova.es as well as the major active social networking websites, where you will find information concerning our society, its membership, scientific partnerships, education courses, masters and conferences, together with other resources and relevant news.
\end{abstract}

Next V International SIRCOVA congress will be held in Valencia: June 2016.

Secretariat SIRCOVA: Ilustre Colegio de Medicos de Valencia, Av/de la Plata 34; 46013 Valencia, Spain.

info@sircova.es www.sircova.es.

\section{About RIG}

The Spanish-Portuguese glaucoma group was founded in 2010 as an informal glaucoma specialist body from our two countries to improve collaboration, to promote and disseminate study data pertaining glaucoma and to ensure the effective delivery of glaucoma prevention, diagnosis, therapy and research throughout the Iberian Peninsula. The group meets each two years when one of the foundational members hosts the scientific meeting, either in Valencia or Porto, as the Reunion Ibérica de Glaucoma/ Reunião Ibérica do Glaucoma (RIG). To date it continues in this light, and in its third edition this growing group will consolidate its role in promoting glaucoma knowledge in our countries. The III RIG has joined the 2014 SIRCOVA congress in Valencia to exchange experiences and to work appropriately on the objectives of efficient and sustainable glaucoma management by taking advantage of the joined organization and to achieve important service quality with a significant reduction in global spending. For more information please keep in touch through:

info@rig.es www.sircova.es

The IV RIG edition will be run in Porto: October 2016.

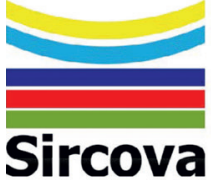

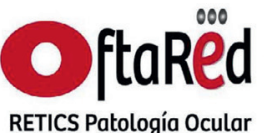

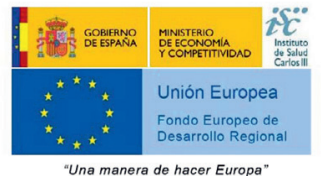

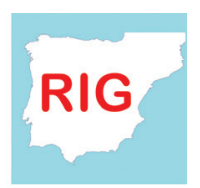

\section{KARGER}

E-Mail karger@karger.com

www.karger.com/ore (c) 2014 S. Karger AG, Basel

$0030-3747 / 14 / 0524-0175 \$ 39.50 / 0$ 


\section{Presidential Greeting}

As a foundational member of the Society of Research in Retina and Visual Sciences (SIRCOVA) and the Iberian Glaucoma Group (RIG), it is my pleasure and my privilege to welcome you all to Valencia for our joined Congress.

Our main purpose is to provide a forum at which a multidisciplinary array of ophthalmologists, scientists, optometrists, students, health care professionals and industry representatives can meet, learn, investigate and create new ideas, the outcomes of which have to be translated to the clinical practice to better manage the ophthalmologic patients.

I encourage all to take advantage of the two Congresses that will highlight the most relevant issues regarding the prevention, diagnosis and treatment of ocular disorders, ranging from basic research to clinical and population studies. These activities will be accomplished in various experts sessions, plenary lectures, monographic symposiums, as well as express free communications ( 3 slides/3 minutes), and other activities that can be discovered in both the SIRCOVA and RIG programs (www.sircova.es).

We specially thank our sponsors: Alcon, Allergan, Angelini, Bausch \& Lomb, Bayer, Bloss, Brudylab, Eglé, Novartis, Sistemas Genómicos, Thea and Topcon for excellent support. Thank you, indeed, to the Spanish Net of Ophthalmic Pathology OFTARED (Instituto de Salud Carlos III, Madrid) for outstanding help and financial support.

I would like to thank the submitters who sent in their works. Selection of the adequacy of the submitted abstracts was performed by the Congress Committees. Therefore, those authors selected for the outstanding quality of their work will be competing for the SIRCOVA or RIG Awards. A panel of international experts will select a winner and two honour mentions for the oral communications, Awards are focused on basic science, clinical science, clinical cases/surgical maneuvers. SIRCOVA also will select the best Doctoral Thesis defended in 2013 for a special Award.

Thank you to all of you for joining us in Valencia for the IV SIRCOVA and III RIG Congresses.

Maria Dolores Pinazo-Durán SIRCOVA President RIG Congress Co-Organizer 


\section{Visual Phenotyping in Mutant Mice Deleted for the Taurine-Transporter Gene}

W. Hadj-Saïd ${ }^{1,2,3}$, N. Froger ${ }^{1,2,3}$, I. Ivkovic ${ }^{1,2,3}$, M. Simonutti ${ }^{1,2,3}$, N. Neveux ${ }^{4,5}$, J.A. Sahel ${ }^{1,2,3}$, S. Picaud ${ }^{1,2,3}$

${ }^{1}$ INSERM, U968, Institut de la Vision, Paris, France; ${ }^{2}$ Sorbonne Universités, UPMC Univ Paris 06, UMR_S 968, Institut de la Vision, Paris, ${ }^{3}$ CNRS, UMR 7210, Institut de la Vision, Paris, ${ }^{4}$ Assistance Publique - Hôpitaux de Paris, Service de Biochimie, Groupe Hospitalier Cochin - Hôtel-Dieu 75014 Paris, ${ }^{5}$ Univ Paris Descartes, Fac. de Pharmacie, Lab. de Nutrition, EA 4466 Paris

Purpose: Taurine depletion was previously shown to induce cone and rod photoreceptor degeneration as reported in knockout mice for the taurine transporter (Tau-T KO). More recently, we described the degeneration of retinal ganglion cells (RGCs) upon taurine depletion induced pharmacologically. To understand further, the implication of taurine in retinal cell survival, we are further investigating Tau-T KO mouse.

Methods: Dosage of plasma, visual functioning tests and histological studies were made in Tau-T KO mice.

Results: A marked depletion of plasma and retinal taurine concentrations was observed in homozygous mice whereas only retinal taurine was reduced in heterozygotes. Homozygotes appeared functionally blind at 9 weeks as indicated by optokinetic response and electroretinogram measurements whereas heterozygotes only showed reduced electroretinogram amplitudes. If photoreceptors were lost in homozygotes at this age, the RGC density appeared normal. No histological modification was apparent in heterozygotes.

Conclusions: In despite of blindness in homozygous mice, the absence of RGC degeneration was attributed to mouse pigmentation in the Tau KO mice because RGC degeneration was related to light stimulation in taurine depleted animals, which were on an albino background. Further studies are needed to demonstrate the synergistic effect of taurine depletion and light to induce RGC degeneration.

\section{Searching for the Cellular Effects of the Imbalance Between the $\omega-6 / \omega-3$ Fatty Acids on the Retinal Cytoarchitecture Using the Fat-1 Transgenic Mice Model}

\author{
C. Marco-Ramírez¹, L. Boscá-Gomar², J. Clariá3 , C. López-Vicario³, \\ J. Escandón ${ }^{4}$, C. Galbis-Estrada ${ }^{1}$, M.D. Pinazo-Durán ${ }^{1}$ \\ ${ }^{1}$ Ophthalmic Research Unit Santiago Grisolia, Valencia, ${ }^{2}$ Instituto \\ de Biomedicina Alberto Sols, Madrid; ${ }^{3}$ IDIBAPS, Barcelona, \\ ${ }^{4}$ Department of Pathology, University Hospital Dr. Peset, \\ Valencia, Spain
}

Purpose: Polyunsaturated fatty acids are essential for the retina, while $\omega-6 / \omega-3$ ratio would change under several conditions (disease, exogenous factors and genetic background). Reports on the resistance to obesity, diabetes, dyslipemia, inflammation and ischemic injury, by means of endogenous $\omega$-3 synthesis, leaded us to utilize the fat- 1 transgenic mice model, genetically manipulated to convert all $\omega-6$ to $\omega-3$, to study the effects of higher endogenous $\omega-3$ availability on the retina.

Material and Methods: Twelve mice (6 months old) were divided into 2 groups: wild-type (wt; $\mathrm{n}=12$ eyes) and fat- 1 transgenic (fat-1; $\mathrm{n}=12$ eyes). Eyeballs were processed for morphologic/morphometric/immunohistochemical techniques using antibodies against synaptophysin, calretinin (CAL), glial fibrillary acidic protein, neurofilaments and Ki67. Statistical analysis was performed using the SPSS 15.0 software.

Results: Similar retinal cytoarchitecture and layering was observed in both groups of mice. Different CAL expression pattern was detected in the fat-1 retinas mainly regarding the retinal ganglion cell and optic fiber layers.

Conclusions: Endogenous $\omega-3$ fatty acids increase doesn't induce morphologic alterations in the transgenic mice retina. Further research is needed to deepen into the cellular and molecular consequences of $\omega-6 / \omega-3$ ratio changes for ocular health.

\section{Glial Retinal Response to Intravitreal Injections \\ J. Di Pierdomenico, D. García Ayuso, M. Jiménez López, M. Agudo Barriuso, M. Vidal Sanz, M.P. Villegas Pérez \\ Departamento de Oftalmología, Facultad de Medicina, Universidad de Murcia, IMIB-Arrixaca, Murcia, Spain}

Purpose: To analyze the effect of intravitreal injections in the microglial and macroglial populations of the rat retina.

Methods: Sprague-Dawley rats were divided in 2 groups. One received a single injection and the other one injection every 7 days (total of 3 injections) of PBS, triamcinolone acetonide (TAC, 
Trigón ${ }^{\circledR}$ ), or anti-rat VEGF. Animals were processed one week later, their retinas dissected as whole-mounts and immunodetected with antibodies against IBA-1, GFAP and Vimentin. Qualitative analysis of the glial populations and quantitative analysis of microglial cells were performed.

Results: An increase in the activation and numbers of IBA- $1^{+}$ cells was observed in all retinas. The retinas injected with PBS or anti-rat VEGF showed GFAP+ astrocytic hypertrophy and the retinas injected with TAC showed also arrow-shaped areas doubly immunoreactive with GFAP and Vimentin that corresponded Müller cells. All the retinas that received three injections showed these arrow-shaped areas also higher numbers of IBA- $1^{+}$cells than the retinas that received one injection.

Conclusion: A single intravitreal injection causes macroglial and microglial activation in the rat retina, but certain characteristics of this reaction depend on the injected substance and the number of injections. Several injections produce a stronger response that a single injection.

\section{Increased Microglia Cell Number and Retraction of Their Processes in Mice Retina Contralateral to Experimental Glaucoma Occurs Beyond GCL}

B. Rojas ${ }^{1,2}$, B.I. Gallego ${ }^{1}$, A.I. Ramírez ${ }^{1,3}$, R. Hoz ${ }^{1,3}$, J.J. Salazar ${ }^{1,3}$, M. Vidal-Sanz $z^{4}$ J.M. Ramírez ${ }^{1,2}$

${ }^{1}$ Inst Invest Oftalmol Ramón Castroviejo, RETICs OFTALRED RD12-0034-0002, UCM, Madrid, ${ }^{2}$ Fac Medicina, UCM, Madrid, ${ }^{3}$ Fac Óptica y Optometría, UCM, Madrid, ${ }^{4}$ Exp Ophthalmol Lab, Department Ophthalmology, College of Medicine, University of Murcia, RETICs OFTALRED RD12-0034-0014, Spain

Purpose: To quantify retinal microglia features of activation 15 days after laser-induced ocular hypertension $(\mathrm{OHT})$ in OHT eyes and their contralateral eyes.

Methods: Adult albino Swiss mice were assigned to two groups: naïve $(n=6)$ and lasered $(n=9)$. Anti-Iba1 was used in retinal whole-mounts to analyse microglial number and the arbour area of each microglial cell.

Results: In comparison with naïve eyes, contralateral eyes and OHT-eyes showed a significant increase in microglial number in the plexiform layers ( $\mathrm{p}<0.05$ and $\mathrm{p}<0.001$ respectively) and in the area of the retina occupied by microglia in the nerve fibreganglion cell layer (NFL-GCL) $(\mathrm{p}<0.01$ and $\mathrm{p}<0.001$ respectively). OHT-eyes exhibited significantly higher values than contralateral eyes for microglial number $(\mathrm{p}<0.001)$ and for the area of the retina occupied by microglial cells in the NFL-GCL $(\mathrm{p}<0.001)$. In comparison with naïve eyes, the microglial arbour area in the plexiform layers was reduced both in OHT-eyes $(\mathrm{p}<0.001)$ and in contralateral untreated eyes ( $\mathrm{p}<0.05$ and $\mathrm{p}<0.001$ respectively).

Conclusions: After 15 days of laser-induced OHT an increase in the number of microglia and retraction of their processes was observed beyond the CGL both in OHT-eyes and in contralateral normotensive eyes. Differences between the two eyes could offer new insights in glaucoma pathophysiology.

\section{Microglia in Mice Retina Contralateral to Experimental Glaucoma Exhibit Qualitative Features of Activation Beyond GCL}

\author{
B.I. Gallego 1, J.J. Salazar ${ }^{1,3}$, A.I. Ramírez ${ }^{1,3}$, B. Rojas ${ }^{1,2}$, R. Hoz $^{1,3}$, \\ M.P. Villegas-Pérez ${ }^{4}$, J.M. Ramírez ${ }^{1,2}$ \\ ${ }^{1}$ Inst Invest Oftalmol Ramón Castroviejo, RETICs OFTARED \\ RD12-0034-0002, UCM, Madrid, ${ }^{2}$ Fac Medicina, UCM, Madrid, \\ ${ }^{3}$ Fac Óptica y Optometría, UCM, Madrid, ${ }^{4}$ Exp Ophthalmol Lab, \\ Department Ophthalmology, College of Medicine, University of \\ Murcia, RETICs OFTARED RD12-0034-0014, Spain
}

Purpose: To study morphological changes and activation features in a model of laser-induced ocular hypertension (OHT) in both OHT-eyes and their contralateral eyes.

Methods: Adult albino Swiss mice were divided into two groups: naive $(n=6)$ and lasered $(n=9)$. Animals were sacrificed two weeks after laser induction. Retinal whole-mounts were immunolabelled with anti-Iba1, anti-CD86, anti-CD68 and antiYm1 and used to analyse the distribution, morphology and activation signs of microglial cells.

Results: In comparison with naive eyes, contralateral eyes and OHT-eyes exhibitited changes in the Iba-1+ cells in all retinal layers. The main differences were: i) presence of ameboid microglia; ii) retraction of cell processes; iii) a higher level of branching; iv) soma displacement; v) reorientation of processes; vi) MHC-II upregulation; vii) increased CD68 immunostaining, and viii) CD86 immunolabelling in ameboid cells. In addition, rounded Iba-1+ CD86+ cells in the NFL-RGC layer and PRL and Ym1+ cells and rod-like microglia in the NFL-RGC were restricted to OHT-eyes.

Conclusions: Several signs of microglial activation were observed in OHT-eyes and contralateral normotensive untreated eyes that extended beyond the GCL. The contralateral eye could provide new insights in glaucoma pathophysiology that could assist the development of future treatments.

\section{Molecular Targets Involved in the Neuroprotective Effect of Tauroursodeoxycholic Acid (TUDCA)}

L. Campello, A. Noailles, V. Ayllón, P. Lax, N. Cuenca,
V. Gómez-Vicente

Universidad de Alicante, Spain

Purpose: The anti-apoptotic properties of the bile constituent tauroursodeoxycholic acid (TUDCA) have been established in models of photoreceptor degeneration. However, the signalling pathways elicited by this compound remain poorly characterized. The aim of this study was to elucidate TUDCA-triggered signalling.

Methods: TUDCA $(800 \mu \mathrm{M})$ was administered to the photoreceptor cell line $661 \mathrm{~W}$ upon induction of apoptosis with the nitric oxide donor sodium nitroprusside $(0.3 \mathrm{mM})$. Viability was assessed by XTT and crystal violet assays. Protein extracts were analyzed using antibody arrays, which allowed for the detection and quantification of well-characterized signalling molecules when phosphorylated or cleaved in specific residues. 
Results: TUDCA increased the viability of $661 \mathrm{~W}$ photoreceptors by reducing SNP-induced apoptosis. Besides, in our experimental conditions, TUDCA played a role in the regulation of key pro-apoptotic proteins phosphorylation. Among the proteins that mediated TUDCA anti-apoptotic action in vitro we identified GSK3beta, HSP27 and the MAPKs p38 and JNK.

Conclusion: Our results shed light on the molecular mechanisms that promote photoreceptor survival and could potentially be extended to the design of novel pharmacological approaches for the treatment of retinal dystrophies.

Support: MINECO-FEDER BFU2012-36845; ISCIII RETICS RD12/0034/0010; ONCE; FUNDALUCE to N. Cuenca; MICINN JCI-2009-05224 to V. Gómez-Vicente; MICINN PLE2009-0111 to V. Ayllón.

\section{Number of Neighbors, a Method to Visualize the Spatial Distribution of Low Population Retinal Cells \\ M. Jiménez-López, F.M. Nadal-Nicolás, C. Galindo-Romero, M. Agudo-Barriuso, M. Avilés-Trigueros, M. Vidal-Sanz \\ Dep. de Oftalmología, Facultad de Medicina, Universidad de Murcia, Instituto Murciano de Investigación Biosanitaria-Virgen de la Arrixaca, Murcia, Spain}

Purpose: To improve the topographic visualization of low population cells in the retina and develop an alternative method to Voronoi diagrams and density maps.

Methods: The k-nearest algorithm with a fixed radius was implemented in Java language, this program loads information from a plain text file where the name of the retina, origin of coordinates and coordinates of each cell is arranged. In a first step a coordinate shift of the origin of coordinates to the optic nerve (ON) location is performed, then for each cell the number of neighbors is calculated as the number of cells closer than the user specified radius.

Results: Calculations are printed into a text file containing the new translated coordinates of all cells, the number of neighbors of each one and its distance to the ON. This information is used with any plotting software to visually represent the results as a multiple scatter plot, where each cell is represented at its X and Y coordinates and coloured according to its number of neighbors within a group class.

Conclusion: This Java program is a reliable alternative to Voronoi and density maps. The program is a write once, run anywhere (WORA) and is available under demand.

\section{Retinal Ganglion Cell Loss and Caspase 3 Activation After Optic Nerve Crush or Transection in Mice}

\author{
M.C. Sánchez-Migallón Carreras, F.J. Valiente-Soriano, \\ F. Nadal-Nicolás, M. Jiménez-López, L. Nieto López, M. Vidal-Sanz, \\ M. Agudo-Barriuso \\ Universidad de Murcia, Murcia, Spain
}

Purpose: To analyze and compare the early loss of mouse retinal ganglion cells (RGCs) and the expression of active caspase 3 after optic nerve transection (ONT) or crush (ONC).

Methods: The left optic nerve of adult albino mice was crushed or sectioned at $0.5 \mathrm{~mm}$ from the optic disk. Animal were sacrificed from 24 hours to 10 days after the lesion, retinas dissected as wholemounts, and Brn3a and active Casp3 (aCasp3) doubly immunodetected to identify surviving and apoptotic RGCs, respectively. The total number of Brn $3 \mathrm{a}^{+} \mathrm{RGCs}$ was automatically quantified and their distribution assessed by isodensity while acasp $3^{+}$RGCs were manual quantified and their distribution analyzed by neighbor maps.

Results: RGC loss after both injuries follows the same course, it is first significant at $3 \mathrm{dpl}$ when approximately $10 \%$ of the original population is lost and by 10 days $70 \%$ of the RGCs have died. acasp $3^{+}$RGCs are observed at $3 \mathrm{dpl}$, their number triples at 4 days and then slowly decreases until 10 days.

Conclusion: RGC death induced by ONT or ONC follows the same temporal trend. RGC loss is first significant 3 days after either injury. RGC death is due, at least in part, to a casp3 dependent mechanism.

\section{Infliximab Reduces Zaprinast-Induced Retinal Degeneration in Cultures of Porcine Retina}

C. Martínez Fernández-De La Cámara, L. Olivares-González,

D. Hervás, D. Salom, J.M. Millán, R. Rodrigo

Instituto de Investigación Sanitaria La Fe, Valencia, Spain

Purpose: Growing evidence suggests that inflammation is involved in the progression of retinitis pigmentosa (RP). The aims of this study were to corroborate the presence of high TNFa concentration in the eye of RP patients and to evaluate whether the blockade of TNFa with Infliximab, a monoclonal TNFa antibody, prevented retinal degeneration induced by PDE6 inhibition in cultures of porcine retina.

Methods: Aqueous humor from $30 \mathrm{RP}$ patients and 13 healthy controls were used to quantify the inflammatory mediators IL-6, TNF $\alpha$, IL- $1 \beta$, IL-10 by ELISA. Porcine retinal explants were exposed to Zaprinast, a PDE6 inhibitor, and/or Infliximab. Cell death was evaluated by TUNEL assay. The number and distribution of caspase- 3 positive cells, indirect poly(ADP) ribose polymerase (PARP) activation and glial fibrillary acidic protein (GFAP) content were visualized by immunolabeling. Antioxidant-oxidant status was also determined.

Results: IL-6 and TNFa were elevated in aqueous humor from $\mathrm{RP}$ patients. Infliximab prevented retinal degeneration and glial activation. Infliximab partially reduced oxidative stress found in retinal explants exposed to Zaprinast. 
Conclusion: Our study suggests that TNFa is playing an important role in cell death in this ex vivo model of retinal degeneration by activating different cell pathways that should be further studied.

\section{Retinal Progenitor Cells Culture in a Medium Supplemented with Lipoic Acid}

L.C. Trachsel Moncho, S. Benlloch Navarro, D.T. Ramírez-Lamelas, R. López Pedrajas, J.M. Soria, M. Miranda

Universidad CEU Cardenal Herrera, Valencia, Spain

Purpose: Several strategies have been proved for cell death amelioration in Retinitis Pigmentosa, such as, neuroprotection and neuroreplacement. Neuroreplacement has been tried by culturing Retinal Progenitor Cells (RPC) forming neurospheres (NS) for a future retinal replacement. This study was designed to prove if LA, an antioxidant, could be used in combination with Fibroblast Growth Factor (FGF) to improve culture conditions of RPC by increasing the growth of NS.

Methods: Primary culture of RPC from postnatal days 0-1 mice retina was performed. We used Neurobasal Medium (Gibco) completed with FGF, antibiotics, B-27 and Glutamine as supplements. Modification of culture media was done by adding LA. NS diameter after 1-4 and 7 days was measured. Differentiation of these NS was achieved by FGF removal. Immunofluorescence assays of Ki-67 and Nestine among others were performed on NS and differentiated RPC.

Results: It was proved that RPC were proliferative and that the NS diameter cultured in LA presence increased.

Conclusion: We suggest LA importance to get a better growing of the RPC, although more studies would be needed to verify that.

This work was partially supported by grants from Fundación Mutua Madrileña and Programa de Investigación CopernicusSantander (PRCEU-UCH 16/12) to MM and Fundación Gangoiti.

\section{Effect and Action Mechanism of Progesterone Administration in rd10 Mouse}

S. Benlloch Navarro, L.C. Trachsel-Moncho, D.T. Ramírez-Lamelas, I. Almansa, J.M. Soria, M. Miranda

Universidad CEU-Cardenal Herrera, Valencia, Spain

Purpose: The aim of this study is to show the neuroprotective role of progesterone (PG) in an animal model of Retinitis Pigmentosa (RP) and elucidate its possible mechanism.

Methods: PG was administered to $\mathrm{rd} 10$ and control mice. Last day of treatment, animals were sacrificed; eyes were enucleated and sectioned or homogenized. Histological evaluation was performed with hematoxilyn-eosin and cell death was assayed using terminal deoxynucleotidyl transferase-mediated dUTP-biotin nick end-labeling (TUNEL). Western blot (WB) of different proteins (iNOS, nNOS, CREB, pCREB, BDNF, TNFa and LC3) and GFAP and nNOS immunohistochemistry were performed. Oxidative stress markers such as glutathione (GSH) and malondialdehyde (MDA) were determined.
To further explore PG mechanism, ARPE-19 PG treated cells were exposed to $\mathrm{H}_{2} \mathrm{O}_{2}$ and cell viability was measured.

Results: After PG treatment amelioration on cell death was observed. No changes were found in WB analyzed proteins, but an increase in GSH/GSSG ratio and a decrease on MDA was observed in PG treated groups. After $\mathrm{H}_{2} \mathrm{O}_{2}$ stress, major viability was detected on ARPE-19 PG treated cells.

Conclusion: Results suggest that progesterone has a protective role in RP, its possible mechanism could be related with its antioxidant ability.

This work was supported by Mutua Madrileña, PRCEU-UCH 16/12 and Fundación Gangoiti.

\section{Characterization of Melanopsin-Containing Ganglion Cells Types in the Human Retina}

\author{
G. Esquiva, P. Lax, N. Cuenca \\ University of Alicante, Alicante, Spain
}

Purpose: To characterize the different types of melanopsin ganglion cells in the human retina, as well as the morphological and quantitative changes associated to aging.

Methods: Human retinas of different ages were whole-mounted and stained against melanopsin using the avidin-biotin complex $(\mathrm{ABC})$. The stratification pattern of each individual labeled ipRGC was manually drawn and digitized. ipRGC morphology was analyzed in detail with the aid of the software NIH Image J.

Results: According to dendritic stratificaction, we have identificated three subtypes of ipRGCs in human retinas: M1 cells, whose dendrites stratified in the S1 stratum; M2 cells, arborizing in the S5 stratum; and bistratified M3 cells, with dendrites occupying both S1 and S5 strata of the IPL. The soma of most of M1 cells were located in the INL, considering these cells as displaced M1. Inmunoreactivity was weaker in M2 than in M1 and M3 cells. Dendritic area and soma size of ipRGCs significantly decreased with aging. Furthermore, mean density of melanopsin-containing cells gradually fell with aging.

Conclusion: The human retina has M1, M2 and M3 melanopsin-expressing ganglion cells, whose soma size, dendritic area and mean density decrease with aging.

Support: MINECO-FEDER (BFU2012-36845), Instituto de Salud Carlos III (RETICS RD12/0034/0010), ONCE and FUNDALUCE.

\section{Expression Analysis of Glaucoma Candidate Genes by 3D Confocal Imaging of Fluorescent Wholemount In-Situ Hybridization (F-WISH) in Zebrafish Embryos}

\section{J.D. Aroca-Aguilar, J.J. Ferre, C. Medina-Trillo, J. Escribano}

Human Molecular Genetics Lab. School of Medicine of Albacete. University of Castilla-La Mancha, Albacete, Spain

Purpose: To analyze spatio-temporal expression patterns of glaucoma candidate genes obtained from massive exome sequencing of a group of primary congenital glaucoma patients. 
Methods: Congenital glaucoma candidate genes with mutations present in compound heterozygosis in primary congenital glaucoma patients and whose embryo expression patterns were unknown were selected. Orthologous zebrafish genes were amplified and cloned, sense and antisense mRNA probes were obtained by RT-PCR and labeled with fluorescent reagent Alexa Fluor 488. High-resolution 3D confocal imaging of fluorescent wholemount in-situ hybridization (F-WISH) in zebrafish embryos at different developmental stages (24 to 96 hours post fecundation) were obtained.

Results: The analysis of the expression patters allowed to characterize its ocular expression in different zebrafish embryologic stages (24-96 hpf). 3D imaging shows abundant expression in anterior segment histological structures that lead to developing aqueous humor draining structures in the zebrafish eye.

Conclusion: The expression patterns support the putative role of the candidate genes in the development of primary congenital glaucoma.

\section{ipRGC Population in Pigmented and Albino Mice}

F.J. Valiente-Soriano, D. García-Ayuso, A. Ortín-Martínez, M. Jiménez-López, M. Agudo-Barriuso, A.A. Vugler, M. Vidal-Sanz

Dep. Oftalmol., Facultad de Medicina, Univ de Murcia, and Instituto Murciano de Invest, Biosanitaria Virgen de la Arrixaca (IMIB-Arrixaca), Murcia, Spain

Purpose: To study the population and distribution of intrinsically photosensitive retinal ganglion cells (ipRGCs) in adult pigmented and albino mice.

Methods: Adult pigmented (C57BL/6) and albino (Swiss) mice RGCs were retrogradely traced from the optic nerve (ON) or from the superior colliculi (SCi). Retinas were immunoreacted against Brn3a and melanopsin.

Results: Pigmented and albino mice have a similar total number of ipRGCs $(1021 \pm 141$ and $962 \pm 169$, respectively). Their distribution is slightly different: while in pigmented mice ipRGCs are more abundant in the temporal retina, in the albino mice ipRGCs are more abundant in the superior retina. All ipRGCs were traced from the $\mathrm{ON}$, except in the retinal rim, where $21 \%$ (pigmented) and 25\% (albino) of ipRGCs were not. Tracing from the SCi shows that $94.5 \%$ (pigmented) and 97.2\% (albino) of the total ipRGCs, project to the SCi, except in the retinal rim, where $26 \%$ (pigmented) and 31\% (albino) of the ipRGCs are not traced. Double immunodetection of melanopsin and Brn3a shows that $1.93 \%$ (pigmented) and $0.78 \%$ (albino) ipRGCs express this transcription factor.

Conclusion: ipRGCs are a well defined subtype of RGCs and represent the $2.5 \%$ and $2.1 \%$ of the pigmented and albino RGC population, respectively.

\section{Cone-Photoreceptor Neuroprotection by Brimonidine, BDNF, PEDF or bFGF in a Novel in Vivo Model of Focal Light Emitting Diode-Induced Phototoxicity in Adult Albino Rats}

\author{
A. Ortín-Martínez, FJ. Valiente-Soriano, D. García-Ayuso, \\ M.I. Jiménez-López, F.M. Nadal-Nicolás, M.P. Villegas-Pérez, \\ M. Vidal-Sanz \\ Dep. Oftalmol, Facultad de Medicina, Instituto Murciano de \\ Investigación Biosanitaria Virgen de la Arrixaca (IMIB), Murcia, \\ Spain
}

Purpose: To investigate the effects of light-emitting diode (LED)-induced phototoxicity (LIP) on cone-photoreceptors and their protection with brimonidine (BMD), brain-derived neurotrophic factor (BDNF), pigment epithelium-derived factor (PEDF), ciliary neurotrophic factor (CNTF) or basic fibroblast growth factor (bFGF).

Methods: Left eyes were exposed to blue LED (10sec, 200lux) and both retinas were assessed using Spectral Domain Optical Coherence Tomography (SD-OCT) and immune-labelled wholemounts for L- and S-opsin. The effects of topical BMD (1\%) or, intravitreal BDNF $(5 \mu \mathrm{g})$, PEDF $(2 \mu \mathrm{g})$, CNTF $(0.4 \mu \mathrm{g})$ or bFGF $(1 \mu \mathrm{g})$ after LIP were assessed at 7 days.

Results: SD-OCT showed damage in a circular region of the superotemporal retina, where retinal thickness decreased from $183.4 \pm 5 \mu \mathrm{m}$ to $114.6 \pm 6 \mu \mathrm{m}(7 \mathrm{~d})$. Wholemounts demonstrated a circular region containing lower numbers of L- and S-cones. Within a circular area ( 1 or $1.3 \mathrm{~mm}$ radius) of the left and its corresponding region of the contralateral-retina, L- or S-cones were $7,118 \pm 842$ or $661 \pm 125$ for the LIP retinas $(n=7)$ and $14,040 \pm$ 1,860 or $2,255 \pm 193$ for their fellow retinas $(n=7)$, respectively. All treatments except CNTF showed significant neuroprotective effects on L- and S-cones.

Conclusions: LIP is a reliable model to study cone-photoreceptor degeneration. Intravitreal BDNF, PEDF or bFGF, or topical BMD afford cone neuroprotection in this model.

\section{Influence of Photoreceptor Degeneration in the Population of Melanopsin ${ }^{+}$and Brn3a ${ }^{+}$RGCs}

\begin{abstract}
D. García Ayuso, J. Di Pierdomenico, N. Cuenca, I. Pinilla, M. Vidal-Sanz, M. Agudo-Barriuso, M.P. Villegas Pérez
\end{abstract}

Dep. Oftalmol. F. de Medicina, Univ de Murcia, Instit. Murciano de Investigación Biosanitaria-Virgen de la Arrixaca (IMIBARRIXACA), Murcia, Spain

Purpose: To document the short and long term effect of photoreceptor loss on the intrinsically-photosensitive Retinal Ganglion Cell (ipRGC) population of the $\mathrm{P} 23 \mathrm{H}-1$ rat retina and the changes in Brn3a expression by these cells.

Methods: $\mathrm{P} 23 \mathrm{H}-1$ rats at post-natal days $(\mathrm{P}) 30(\mathrm{n}=6), \mathrm{P} 180$ $(\mathrm{n}=6)$ and P540 $(\mathrm{n}=5)$ and Sprague-Dawley $(\mathrm{SD})$ rats at $\mathrm{P} 30(\mathrm{n}=$ 5) and P540 ( $n=5)$ were used. The retinas were dissected as whole-mounts and processed with antibodies against Brn3a and 
melanopsin. The numbers of Brn3a ${ }^{+}$RGCs and melanopsin ${ }^{+}$RGCs and the co-localization of both markers were analyzed.

Results: The total number of Brn $3 \mathrm{a}^{+} \mathrm{RGCs}$ in $\mathrm{P} 23 \mathrm{H}-1$ rats was already smaller at P30 and decreased also with age, in accordance with our previously published data (García-Ayuso et al., Exp. Eye Res., 2010). In these animals, there was also a significant decrease with age of the population of melanopsin ${ }^{+}$RGCs. Co-localization analysis showed that in SD rats only $0.27 \%$ of the $\mathrm{m}^{+} \mathrm{RGCs}$ were also $\mathrm{Brn}^{2} \mathrm{a}^{+}$. In $\mathrm{P} 23 \mathrm{H}-1$ rats, however, the percentages of $\mathrm{m}^{+} \mathrm{RGCs}$ that were also ${\mathrm{Brn} 3 \mathrm{a}^{+}}^{+}$were higher and increased with age, reaching $10.65 \%$ at $\mathrm{P} 540$.

Conclusion: Photoreceptor degeneration causes a decrease of the ipRGC population and an increased pattern of co-expression of melanopsin and Brn3a by these cells.

\section{Effect of Hypoxia in Neonatal Pig Retina and Superior Colliculus}

N. Ruzafa, C. Rey-Santano, V. Mielgo, D. Rodríguez, E. Vecino

Dep. Biología Celular e Histología, Univ. País Vasco UPV-EHU, Bilbao, Spain

Purpose: To study the effect of mild hypoxia to neonatal pig retina and brain. We analyze the survival of neurons from the superior colliculus (SC) as well as retinal ganglion cells (RGCs) and the morphological changes in astrocytes in the retina.

Methods: We induced hypoxia to neonatal pigs by decreasing oxygen levels to $12-14 \%$ for 120 minutes followed by 240 minutes of re-oxygenation. RGCs were quantified using Brn3a marker and cell death in the SC with caspase- 3 marker. Morphometric analysis of the retinal astrocytes labeled with GFAP was performance with a program specially designed for that purpose.

Results: We found an increase of $37.63 \%$ in the number of caspase- 3 positive cells in the SC in the hypoxic pigs respect to the control ones; this increase was more evident in the stratum zonale $(56.93 \%)$. We did not find significant changes in the survival of RGC. Hypoxia did not induce significant changes in the morphology of the retinal astrocytes at this time point.

Conclusion: The mild hypoxia induced to the neonatal pig do not affect to the retina but the brain was affected with an increase of cell death in the superior colliculus.

Supported by Grupos Consolidados del Gobierno Vasco (IT437-10).

\section{Absence of Toll-Like Receptor 4 (TLR4) Alters the Structure and Function of the Mouse Retina}

P. Lax, V. Gómez-Vicente, A. Noailles, E. de Juan, V. Maneu, N. Cuenca

Universidad de Alicante, Spain

Purpose: The role of TLRs in proliferation, survival and function of retinal cells remains uncertain. Here we assess the role of TLR4 in the structure and function of the mouse retina.

Methods: Retinal responsiveness and visual acuity were evaluated by electroretinography and optomotor test, respectively, in
TLR4-/- knockout and wild type C57BL/6J mice at P20, P30 and P60. Retinal structure was also assessed at P30 in both experimental models by immunohistochemical techniques and confocal microscopy.

Results: ERG recordings showed impairment of retinal responsiveness in TLR4-/- mice, as compared to wild type animals. The scotopic a- and b-waves were significantly lower in TLR4defective mice than in wild type animals at all ages tested (P20, P30 and P60) (ANOVA, p < 0.05). Visual acuity was also lower in TLR4-/- compared to C57BL/6J mice, with significant differences at P60 (ANOVA, p < 0.05). Vertical retinal sections from P30 TLR4-/- mice showed lower density of bipolar cells and less synaptic contacts between photoreceptors and bipolar cells (ANOVA, $\mathrm{p}<0.05$ in both cases) than in the wild type.

Conclusion: Absence of the innate immune receptor TLR4 alters the structure and impairs the function in the mouse retina.

Support: MINECO-FEDER (BFU2012-36845), Instituto de Salud Carlos III (RETICS RD12/0034/0010), ONCE, FUNDALUCE.

\section{Permanent Microglial Activation During All Stages of Retinal Degeneration}

V. Maneu, A. Noailles, L. Campello, V. Gómez-Vicente, P. Lax, N. Cuenca

University of Alicante, Spain

Purpose: The activation of microglia has been demonstrated in association with several neurodegenerative diseases. The main objective of this work was to study the temporal progression of microglial activation in a model of retinal degeneration, in order to correlate it with visual impairment.

Methods: Microglial activation was analyzed in homozygous $\mathrm{P} 23 \mathrm{H}$ line 3 and Sprague-Dawley rats (control) at 1, 2, 3, 4, 6 and 12 moths of age by flow cytometry, using CD11b, CD45 and MHC class II cell-surface markers. Immunohistochemical assays were also performed.

Results: Activated microglia were detected by flow cytometry at all ages in the retinas of $\mathrm{P} 23 \mathrm{H}$ rats, while no signs of microglial activation were observed in the retinas of Sprague-Dawley rats. Activated microglia were minimal at 1 month of age and maximal at 12 months, with an apparent plateau from 2 to 6 months of age. The immunohistochemical analysis allowed the identification of microglial cells with amoeboid morphology and increased MHCII expression in $\mathrm{P} 23 \mathrm{H}$ rat retinas.

Conclusion: Increased microglial density and activation persists in the retina, even after photoreceptor death, in a model of retinal neurodegeneration.

Support: MINECO-FEDER BFU2012-36845; ISCIII RETICS RD12/0034/0010; ONCE; FUNDALUCE; Fundación Jesús de Gangoiti Barrera. 


\section{Norgestrel is Able to Protect Retinal Cytoarchitecture and Synaptic Connectivity in a Retinitis Pigmentosa Model}

\author{
L. Fernández-Sánchez, V. Gómez-Vicente, G. Groeger, F. Doonan, \\ T.G. Cotter, N. Cuenca \\ Universidad de Alicante, Alicante, Spain
}

Purpose: Attenuation of photoreceptor apoptosis in animal models of retinal degeneration has been achieved by treatment with norgestrel, a synthetic progestin commonly used in contraception. In this study we evaluate norgestrel ability to halt retinal cytoarchitecture disruption and synaptic connectivity impairment in the $r d 10$ mouse.

Methods: Norgestrel (100 mg/kg ip) or vehicle were administered to $r d 10$ mice from P18 to P40 on alternate days. The eyes were fixed and vertical cryosections, stained with antibodies for different retinal cell markers, were visualized by confocal microscopy.

Results: Compared to vehicle-treated animals, retinas from norgestrel-treated $r d 10$ mice showed good preservation of cone photoreceptor morphology, especially in the peripheral retina, as revealed by immunostaining with gamma-transducin. Additionally, calbindin, GNB3 and PKC-alpha labeling revealed improved horizontal and bipolar cell morphology. Stronger immunoreactivity for the synaptic ribbon components CtBP2 and bassoon, and the synaptic vesicle glycoprotein synaptophysin was also observed.

Conclusion: Our results prove that norgestrel delays retinal remodeling and synaptic contact loss associated to photoreceptor degeneration, indicating it may be a good therapeutic candidate for the treatment of retinitis pigmentosa and related diseases.

Support: MINECO-FEDER BFU2012-36845; ISCIII RETICS RD12/0034/0010; ONCE; FUNDALUCE to N. Cuenca; HRB^ HRA_POR/2011/2 and Fighting Blindness Ireland to T.G. Cotter; MICINN JCI-2009-05224 to V. Gómez-Vicente.

\section{Effect of a Series of Hyaluronic Acid Intracamerular Injections on the Rat Retinal Ganglion Cells and Optic Axons Characteristics}

\author{
A. Mayordomo Febrer' ${ }^{1}$, M. López-Murcia' , C. Marco-Ramírez², \\ M. Avilés-Triguero ${ }^{3}$, N. Cuenca ${ }^{4}$, M.I. López Gálvez ${ }^{5}$, \\ M.D. Pinazo-Durán ${ }^{2}$ \\ ${ }^{1}$ University Cardenal Herrera CEU, Valencia, ${ }^{2}$ Ophthalmic \\ Research Unit Santiago Grisolia, Valencia, ${ }^{3}$ University of Murcia, \\ ${ }^{4}$ University of Alicante, ${ }^{5}$ IOBA-Universidad de Valladolid, Spain
}

Purpose: To set up an experimental chronic glaucoma model in rats.

Methods: Thirty one juvenile male Wistar rats were used. A set of experiments were designed through ten consecutive weeks. Either hyaluronic acid/balanced salt solution was injected into the anterior chamber of the rat left $(\mathrm{LE} ; \mathrm{n}=25)$ or right eye $(\mathrm{RE} ; \mathrm{n}=$ 25 ) once a week. Five of these rats were later intracraneally injected with Fluorogold ${ }^{\circledR}$ to detect retinal ganglion cell (RGC) and
Brn3a immunolabeling in their whole mount retinas. Nine of our experimental rats were used to morphologic and morphometric analyses in their retina/optic nerve cross-sections. Six non-operated rats were used as the controls. Statistics were done by the SPSS 15.0 program.

Results: The LE displayed significantly lower Fluorogold traced RGC and Brn3a immunostaining than the RE $(\mathrm{p}<0.001)$. Reduced retinal thickness and optic nerve cross-sectional area were found in the LE respect to the sham-operated eyes and the controls.

Conclusions: The sustained intraocular hypertension induced by the intracameral hyaluronic acid injections in the rat eyes lead to a series of structural, functional and molecular changes that can be extrapolated to those described for the human glaucomatous optic neuropathy.

Support: Murcia/Alicante/Valladolid/Valencia collaborative groups of 'OFTARED': RETICS RD12-0034-003 - 0004/0011/ 0013/0014.

\section{Comprehensive Analysis of microRNA in Tears}

C. Campos-Borges², V. Zanón-Moreno ${ }^{1}$, C. Galbis-Estrada ${ }^{1}$,

C. Marco-Ramírez' ${ }^{1}$ M.l. López Gálvez ${ }^{2}$, J. Salgado-Borges², M.D. Pinazo-Durán ${ }^{1}$

${ }^{1}$ Ophthalmic Research Unit Santiago Grisolia, Valencia, ${ }^{2} \mathrm{Hospital}$ de Porto, Portugal; ${ }^{2}$ IOBA-Universidad de Valladolid, Spain

Purpose: Recent reports show that tears can regulate epithelial microRNA expression in order to regulate genes involved in ocular surface immunity. Main goal of this study was to set up a tear extraction protocol and molecular analysis that permits the extraction of micro RNAs using a single process to be translated to the clinical practice.

Methods: Reflex tears were collected from the inferior meniscus of both eyes by gentle rubbish with a Pasteur micropipette. 15-20 microliters were obtained and immediately stored in microeppendorfs at $-80^{\circ} \mathrm{C}$. Tear samples were extracted using micro RNA Kits. Data were normalized and compared between groups by specific software.

Results: The resulting extracts containing microRNA then underwent standard analysis to compare between patients. By carrying out a comparison between the amounts of micro RNA, it could be seen that the tear expression clearly vary between samples.

Conclusions: With these protocols and the results obtained it is presumably to have an important impact in ophthalmology and ophthalmic research for studying individual cases or specific ocular diseases.

Support: FIS. PI13/00480 and RETICS-OFTARED Groups RD12/0034/0008-RD12/0034/0001. 


\section{Identification of Genes Involved in Type 2 Diabetes Mellitus in a Mediterranean Population}

K. Shoaie Nia, B. Vivar-Llopis, C. Galbis-Estrada, C. Marco-Ramírez, M.D. Pinazo-Durán, V.Zanón-Moreno

Ophthalmic Research Unit Santiago Grisolia, Valencia, Spain

Purpose: To analyze the expression of TMCO1, TP53, SLC23A2, MMACHC, MMADHC and MMP9 genes in relation to type 2 diabetes mellitus (T2DM).

Methods: We recruited 70 suitable participants distributed into two groups: 1) T2DMG ( $\mathrm{n}=40)$ and 2) controls (GC; $\mathrm{n}=30)$ from the Mediterranean area of Valencia Spain. Ananmesis, personal/ familial background, lifestyle data and blood samples collection were done. The RNA was extracted and converted to cDNA using a commercial kit (Applied Biosystems). Gene expression was analyzed by qPCR using TaqMan assays and 7900HT Sequence Detection System. Statistics was performed by the SPSS 15.0 program.

Results: Significantly lower expression (fold change) of SLC23A2, MMACHC, MMADHC and MMP9 (0.83 \pm 0.40 vs $1.00 \pm 0.15, \mathrm{p}=0.021 ; 0.73 \pm 0.28$ vs $1.00 \pm 0.20, \mathrm{p}=0.004 ; 0.80 \pm$ 0.51 vs $1.00 \pm 0.10, p=0.012 ; 0.81 \pm 0.40$ vs $1.00 \pm 0.22, p=0.018$; respectively) and a significantly higher expression (fold change) of TP53 gene $(2.00 \pm 0.31$ vs $1.00 \pm 0.12, \mathrm{p}=0.008)$ was observed in T2DMG compared with the CG.

Conclusion: These data suggest that the whole blood expression level of these genes may be specific for T2DM and can be used as biomarker genes associated with susceptibility and/or therapeutic response to vitreoretinal complications.

\section{Micro RNAs in Tears Correlates with Pivotal Risk Factors for Diabetic Retinopathy}

M.D. Pinazo-Durán' , J.J. García-Medina², D. Galarreta',
C. Marco-Ramírez', C. Galbis-Estrada', R. Gallego-Pinazo',
M.I. López-Gálvez
'Ophthalmic Research Unit 'Santiago Grisolía', Valencia,
${ }^{2}$ Hospital general Univ. Reina Sofía, ${ }^{3}$ IOBA-Universidad de
Valladolid, Murcia, Spain

Purpose: Diabetic Retinopathy (DR) it is the third leading cause of blindness worldwide, but genetics and epigenetics of DR remains unknown. Recently, miRNAs have been identified/quantified in various body fluids and their aberrant expression associated with various diseases.

Methods: A pilot study was designed to investigate the miRNAs expression in tears. Participants were divided into: 1 ) type 2 diabetics (T2DMG) with RD $(\mathrm{n}=18), 2)$ T2DMG without retinopathy $(\mathrm{n}=$ 12 ) and 3 ) healthy controls $(n=24)$. Anamnesis to define patient characteristics and risk factors, ophthalmic examination and collecting tears from both eyes was done to all groups. Tears miRNA extraction was done by specific kits and multiple analyses by specific software was carried out. Data were processed by the SPSS 15.0 program to integrate molecular/demographic/ophthalmic variables.

Results: Significantly different miRNAs expression was found in tears from the GC vs the T2DMG $(\mathrm{p}<0.001)$. The T2DMG with
RD and familial history of hypertension (HTA) had significantly higher values $(p<0.001)$ of miRNAs than those without it. The regular exercise induced a decreased expression of miRNAs in tears of T2DMG with and without DR $(\mathrm{p}<0.01)$.

Conclusions: miRNAs exhibit differential expression in tears from diabetics that correlated with pivotal risk factors for the progression of DR.

Support: FIS. PI13/00480 and RETICS-OFTARED Groups RD12/0034/0008-RD12/0034/0001.

\section{Type 2 Diabetes Mellitus Exhibits a Tear-Specific Expression Pattern of miRNAs}

\author{
V.Zanón-Moreno' , C. Marco-Ramírez'1 , M.I. López-Galvez², \\ D. Galarreta ${ }^{2}$, J.J. García-Medina ${ }^{3}$, R. Gallego-Pinazo', \\ M.D. Pinazo-Durán ${ }^{1}$ \\ 'Ophthalmic Research Unit 'Santiago Grisolía', Valencia, \\ ${ }^{2}$ IOBA-Universidad de Valladolid, ${ }^{3}$ Hospital General Univ. Reina \\ Sofía, Murcia, Spain
}

Purpose: Type 2 diabetes mellitus (T2DM) pandemic is a major public health problem. Genetics of T2DM remains unresolved. Altered miRNAs expression has been linked to a high variety of diseases.

Methods: We designed this study to evaluate the expression of miRNAs in tears of T2DM patients, according to the inclusion/ exclusion criteria, subdivided into 2 groups: 1$)$ T2DMG $(n=30)$ and 2) healthy controls (CG; $n=24)$. Data were collected from clinical history, ophthalmic examination and tears collected from inferior meniscus of both eyes, that were frozen until processing. Tears miRNA extraction was done by specific kits that were quantified by a Nanodrop ${ }^{\circledR}$ with multiple analyses by specific software. Statistics were performed by the SPSS 15.0 program.

Results: Median age of T2DMG was $68 \pm 12$ vs $43 \pm 21$ years in the CG. It was found a significantly higher miRNAs expression in tears from the T2DMG vs the CG $(9.02 \pm 3.08 v s 6.85 \pm 3.58 \mathrm{ng} /$ microL; $\mathrm{p}=0.022$ ).

Conclusions: Based on our 'in vivo' study we conclude that diabetics showed a differential expression pattern of miRNAs in tears. Identifying the specific miRNAs involved in T2DM and retinopathy, we will design new therapeutic strategies for better managing the eyes and vision.

Support: FIS. PI13/00480 and RETICS-OFTARED Groups RD12/0034/0008-RD12/0034/0001.

\section{Differential Metabolomic Profile in Tear Samples Related to Ocular Surface Dysfunction}

\section{Galbis-Estrada, B. Vivar-Llopis, J. Morales, V. Zanón-Moreno, C. Marco-Ramírez, M. Díaz-Llopis, M.D. Pinazo-Durán}

Ophthalmic Research Unit 'Santiago Grisolía', Valencia, Spain

Purpose: Improvements in analytical techniques lead us to propose new approaches for studying the human tear composition and diagnosis/treatment of eye surface diseases (ESD). Me- 
tabolomics was used to analyze reflex tears composition from ESD patients.

Material and Methods: Ninety participants were divided into 2 groups: 1 ) patients with ESD (ESDG, $\mathrm{n}=55)$ and 2) healthy subjects $(\mathrm{GC}, \mathrm{n}=35)$. The ESDG was subdivided into 2 subgroups according to the severity of symptoms [mild-moderate $(n=22)$, or moderate $(n=33)]$. Samples of the lower tear meniscus from both eyes were collected using a Pasteur pipette, which were stored at $-80^{\circ} \mathrm{C}$ until analysis. Metabolomic profile was obtained by 1 proton Nuclear Magnetic Resonance (1HRNM). Data were processed and transferred to MATLAB for further chemometric analysis.

Results: Mean age of participants was $44 \pm 14.5$ years, and females-males distribution was $75 \%-58 \%$ in the ESDG vs $25 \%-42$ in the GC. Main significant differences were found in the levels of cholesterol, $\mathrm{N}$-acetylglucosamine, glutamate, creatine, amino-nbutyrate, choline, acetylcholine, arginine, phosphoethanolamine, glucose, and phenylalanine. Differential metabolic profile helped to discriminate also between subgroups of severity.

Conclusion: The ESD induced metabolic changes in tears identified by $1 \mathrm{HNMR}$ may be useful in understanding the pathogenesis and to design new biomarkers for eye disorders.

In collaboration with the Madrid Group of OFTARED. RETICS RD12-0034-0003.

\section{Two Methods to Trace Rat RGCs with Fluorogold: Around the Optic Nerve without Axotomy and by a Single Stereotactic Injection}

\author{
F.M. Nadal-Nicolás, M. Salinas-Navarro, M. Jiménez-López, \\ L. Nieto-López, M.C. Sánchez-Migallón, M. Vidal-Sanz, \\ M. Agudo-Barriuso \\ Instituto Murciano de Investigación Biosanitaria-Hospital \\ Clínico Universitario Virgen de la Arrixaca, Dto Oftalmología, \\ F. Medicina, Univ. de Murcia, Spain
}

Purpose: To trace retinal ganglion cells (RGCs) with Fluorogold (FG) from the optic nerve without injury, or by a single injection into optic tract.

Methods: To trace from the optic nerve (ON), the meninges were opened longitudinally along their dorsal aspect. The ON was wrapped with a stripe of gelatine sponge soaked in FG and the retinas analyzed 3 days later. To trace from the optic tract a single injection of FG (1?l) was made into it using Paxinos \& Watson, (1998) coordinates. Retinas were analyzed 7 days later. As tracing control, some retinas were traced from both superior colliculi using standardized procedures. As control of RGC damage, Brn3a was immunodetected. In all retinas the total number of traced and Brn3a positive RGCs was automatically quantified and their distribution visualized by isodensity maps.

Results: There was no significant difference in the total number and retinal topography of FG+RGCs and Brn3a+RGCs among the three methods. This indicates that tracing from the $\mathrm{ON}$ or the optic tract labels the whole RGC population and that at the survival intervals after the tracing, no RGC death was observed.

Conclusion: These two methods are relatively easy, reliable, effective and straightforward to trace with FG rat RGCs.

\section{Activated Microglia is Present in Retina Contralateral to Experimental Glaucoma but Rod Microglia is Restricted to Eyes with Ocular Hypertension}

\author{
R. De Hoz ${ }^{1,3}$, B.I. Gallego ; A.I. Ramírez ${ }^{1,3}$, J.J. Salazar ${ }^{1,3}$, B. Rojas ${ }^{1,2}$, \\ M. Aviles-Trigueros ${ }^{4}$, J.M. Ramírez ${ }^{1,2}$ \\ ${ }^{1}$ Inst Invest Oftalmol Ramón Castroviejo, RETICs OFTALRED \\ RD12-0034-002, UCM, Madrid, ${ }^{2}$ Fac Medicina, UCM, Madrid, \\ ${ }^{3}$ Fac Óptica y Optometría, UCM, Madrid, ${ }^{4}$ Exp Ophthalmol Lab, \\ Dep Ophthal, College of Medicine, Univ. Murcia, Spain
}

Purpose: The aim of this study was to analyse the presence of rod microglia in a model of unilateral laser-induced ocular hypertension (OHT).

Methods: Adult albino Swiss mice were divided into two groups: naïve (age-matched control; $n=6)$ and lasered $(n=6)$. Retinal whole-mounts were analysed by immunostaining using antibodies against Iba1, MHC-II, ED1 and NF-200.

Results: In contrast with naïve and contralateral eyes only $\mathrm{OHT}$-eyes had rod microglia. Rod microglia lay parallel and close to the axons and were related to retinal ganglion cells (RGCs) showing signs of degeneration (NF-200+RGCs). In some instances, rod microglia processes surrounded the soma and proximal dendrites of NF-200+RGCs and penetrated the underlying RGC layer and inner plexiform layer. MHC-II was up-regulated in microglia in both contralateral and OHT-eyes. However, only in OHT-eyes did rod microglia show ED1 staining throughout the nerve fibre layer. Three stages of rod microglia were detected, in relation to morphological features and to ED1 immunostaining patterns.

Conclusions: After 15 days of unilateral laser-induced OHT, rod microglia was restricted to eyes with OHT and degenerated NF-200+RGCs but absent in contralateral eyes. Macrophage features of rod-like microglia observed here support the hypothesis that these cells could be remodelling disrupted synaptic circuitry.

\section{The Use of an Antioxidant Combination Therapy for Retinitis Pigmentosa}

D.T. Lamelas, S. Benlloch Navarro, L.C. Irachsel Moncho, M. Miranda

Universidad CEU Cardenal Herrera, Valencia, Spain

Purpose: Retinitis pigmentosa (RP) is an inherited retinal disorder that causes progressive photoreceptor cell loss. The purpose of this study was to asses if the use of an antioxidant combination therapy with progesterone and lipoic acid could preserve the viability of photoreceptors in a model of retinal degeneration, providing better results than each of them separately.

Methods: Progesterone (100 mg/kg), Lipoic acid (100 mg/Kg) or a combination of both, were administered orally to $\mathrm{rd} 1$ mice at different postnatal (PN) days. On PN11, eyes were enucleated and sectioned. Histological evaluation was performed using in situ terminal deoxynucleotidyl transferase-mediated dUTP-biotin nick end-labeling (TUNEL) assay.

Results: The combination of progesterone and lipoic acid administration improved cell survival and showed better photoreceptor protection effect than when administered alone. 
Conclusion: Our study show that the use of two substances such as progesterone (that it is known to inhibit lipid peroxidaton) and lipoic acid (a well known antioxidant) has a protective role in $\mathrm{RP}$, however further studies are needed to confirm these results.

This work was supported by grants from Fundación Mutua Madrileña, Programa de Investigación Copernicus-Santander (PRCEU-UCH 16/12) and Fundación Gangoiti.

\section{Signaling Pathways in the Degenerating Retina of rd10 Mice}

V. Gómez-Vicente, L. Campello, V. Ayllón, P. Lax, A. Angulo, N. Cuenca

Universidad de Alicante, Alicante, Spain

Purpose: To provide an overview of pro- and anti-apoptotic signaling pathways activated at the early stages of retinal degeneration.

Methods: Animals used in this study were treated in accordance to the ARVO statement for the use of animals in ophthalmic and vision research. $r d 10$, an autosomal recessive retinitis pigmentosa model, and wild type (C57BL/6) mice were sacrificed on P22. Retinal protein extracts were obtained and analyzed using antibody arrays that allow for the detection and quantification of well-characterized signaling molecules when phosphorylated or cleaved in specific residues.

Results: Proteins that showed increased phosphorylation in the $r d 10$ retina included the mitogen-activated protein kinases ERK1/2 and JNK, the transcription factor STAT3, the energy balance regulators AMPK and MTOR, the chaperone HSP27 or the glycogen synthase kinase 3-beta (GSK3b), among others. Interestingly, the apoptotic mediators BAD, caspase- 3 and PARP were inhibited in the $\mathrm{P} 22$ rd10 retina.

Conclusion: Our data reveal activation of pro-survival mechanisms at the initial phase of retinal degeneration in the $r d 10$ mouse.

Funding: MINECO-FEDER BFU2012-36845; ISCIII RETICS RD12/0034/0010; ONCE; FUNDALUCE to N. Cuenca; MICINN JCI-2009-05224 to V. Gómez-Vicente; MICINN PLE2009-0111 to V. Ayllón.

\section{Electrophysiological Light Responses in Müller Derived Cell Line}

A. Gracia Llorente, J. Vicente-Tejedor, V. Gómez-Vicente, N. Cuenca, P. De la Villa

Dep de Biología de Sistemas. Univ de Alcalá, Alcalá de Henares, Madrid, Spain

Purpose: A new murine retina cell line (MU-PH1) has been functionally characterized. These derived Müller cells may have the potential to differentiate to a photosensitive cells and express different types of proteins implied in phototransduction cascade. In order to evaluate how light could induce electrophysiological responses in these cells, we have undertaken electrophysiological recordings.
Methods: Ionic currents mediated by potassium channels were recorded under whole cell configuration - patch clamp technique in MU-PH1 cultured cells. Currents were activated by voltage pulse and ramps protocols in two different experimental conditions: room $\left(\approx 22^{\circ} \mathrm{C}\right)$ and $37^{\circ} \mathrm{C}$ temperature. Light responses were recorded from MU-PH1 cells in absolute darkness and after $5 \mathrm{~min}$ of light stimulation. Recovery of light responses was tested along $45 \mathrm{~min}$ of time recovery in darkness.

Results: Light stimulation induces the decrease in $\mathrm{K}^{+}$current in MU-PH1 cells and fully recover after light off. Significant differences were observed under distinct temperature conditions.

Conclusion: We conclude that the expression of phototransduction cascade proteins in MU-PH1 cell is functionally associated to the light induced decrease in $\mathrm{K}^{+}$current amplitude. This new type of photosensitive cell could be possibly used in future for transplant experiments on animal models of retinal degeneration.

\section{Intravitreal Dexamethasone Implant for Treatment of Persistent Diabetic Macular Edema}

\section{Z. Del Barrio, S. Mediero, J. García-Martínez, M. Asencio-Durán, F. Armadá-Maresca}

La Paz University Hospital, Madrid, Spain

Purpose: To evaluate the effectiveness of a single dexamethasone intravitreal implant (Ozurdex ${ }^{\circledR}$; Allergan) over a 12 month period in patients with persistent diabetic macular edema (DME).

Methods: Retrospective review set out to evaluate the effectiveness of a single dexamethasone intravitreal implant over 12 months in 43 eyes with chronic DME.

Results: Treatment with the dexamethasone implant produced a significant improvement in the mean best-corrected visual acuity, increasing from 0.16 at baseline to $0.21(\mathrm{p}=0.0002)$ at month $1,0.19$ at month 5 and $0.19(\mathrm{p}=0.0261)$ at 12 month. Significant improvement in the mean Central Subfield Thickness was also seen 1 and 5 months after treatment with the dexamethasone implant, improving from the baseline value of 499.2 to 309.9 ( $p<$ $0.0001)$ at month 1 and $380.1(\mathrm{p}<0.0001)$ at month 5 . The mean Average Central Thickness values improved from the mean basal of 357.1, to a value of $312.5(\mathrm{p}<0.0001)$ at month 1 , of 322.6 ( $\mathrm{p}<$ $0.0001)$ at month 5 , and of $322.6(p=0.0001)$ at month 12 . No serious adverse events were detected.

Conclusions: The current study suggests that a single dexamethasone implant is a favorable treatment option for patients with DME to improve visual acuity and reduce macular edema. 


\section{Opticocilliary Shunt Vessels in Angle-Closure Glaucoma}

F.J. Hernández Martínez, P. Piñas García, L. Castillón Torre, N. Aznarez López, M.E. Tena Sempere, S. López Aramburu

Hospital San Juan de Dios del Aljarafe, Sevilla, Spain

Purpose: We describe the differential diagnosis by means of images on the presence of opticocilliary shunt vessels in a case of chronic angle-closure glaucoma.

Methods: A case clinic report.

Results: Patient with visual loss of the right eye during a 2 -month period with no associated symptoms. The intraocular pressure of the right eye was $60 \mathrm{~mm} \mathrm{Hg}$; closed anterior chamber angle; transparent cornea; relative afferent pupillary defect; Bestcorrected visual acuity: light perception. Vertical optic nerve cupping: 0.95 with concentric thinning of the neural ring. Fluorescein angiography presented classic signs of optociliary shunts. No secondary causes of the shunts were encountered (cerebral and orbital magnetic resonance imaging, carotid Echo-Doppler).

Conclusion: In the case we present there is an association between the optocilliary shunt and the maintained subacute angleclosure. A possible mechanism of action is the venous insufficiency secondary to the hypertensive neural damage.

\section{Prognostic OCT Based Classification for Myopic Choroidal Neovascularisation}

\section{Pascual-Camps, R. Dolz-Marco, M. Andreu-Fenoll, R. Gallego-Pinazo \\ Hospital Universitario y Politécnico La Fe, Valencia, Spain}

Purpose: To analyze the prognostic value of an optical coherence tomography (OCT) based anatomic classification of myopic choroidal neovascularizacion ( $\mathrm{mCNV})$.

Methods: Retrospective case series of consecutive patients with $\mathrm{mCNV}$ treated with intravitreal injections of ranibizumab into a 'pro re nata' dosing strategy. Cases were classified according to the OCT into type 1 (complete retinal pigment epithelium (RPE) envelopement), type 2 (CNV on top of the RPE), or mixed lesions.

Results: 90 eyes of 71 patients (51 female; mean age: 59.04 years) were included. Overall the mean visual acuity (VA) improved from 0.35 to 0.43 ( $\mathrm{p}<0.001$ ). Type $1 \mathrm{mCNV}$ showed significant VA gain $(\mathrm{p}<0.001)$, whereas type 2 and mixed $\mathrm{mCNV}$ did $\operatorname{not}(\mathrm{p}=0.24$ and $\mathrm{p}=0.06)$.

Overall the mean number of injections administered per patient was 6.58 throughout a mean follow-up of 33.49 months. Type $1 \mathrm{mCNV}$ needed a mean number of 5.00 injections, while type 2 mCNV needed a mean number of 9.21 injections.

Conclusions: Type $1 \mathrm{mCNV}$ appears to have a better visual outcome and to need a smaller number of injections. The OCT classification of mCNV may be of great prognostic value. Further studies are warranted in order to validate this novel approach.

\section{Comparisons of Macular Structural/Functional Parameters as Measured by SD-OCT and Automated Perimetry Between Healthy Subjects and Early Glaucomatous Patients

\author{
M. Tudela-Molino, J. Fares-Valdivia, P. Sobrado-Calvo, \\ M.P. Villegas-Pérez
} \\ M.B. Rodríguez-Cavas, J.J. García-Medina, E. Rician Rodica, \\ Hospital General Universitario Reina Sofía, Murcia, Spain}

Purpose: To compare different macula-related parameters between healthy subjects and patients with early open-angle glaucoma and study the intragroup relationships among these parameters.

Methods: 47 patients were selected (22 were healthy subjects and 25 patients suffering from early open-angle glaucoma). All of them underwent a complete ocular examination, optic coherence tomography (SD-OCT, Spectralis) and automated perimetry (Humphrey). Ganglion cell layer + inner plexiform (GCL+IP) thicknesses, temporal peripapillary retinal nerve fiber layer (RNFL) thickness, foveal sensitivity and the four-innest points' sensitivities in the 10-2 visual field were measured.

Results: Macular GCL+IP thicknesses and visual field sensitivities were significantly different between healthy subjects and early glaucomatous patients. However the peripaIPllary inferior clock hours (7 and 8 hours) thicknesses, theoretically dependent on inferior macular regions, did not show any difference between both groups. Otherwise the correlations between GCL+IP and RNFL were different between healthy and glaucomatous subjects. Additionally correlations between GCL+IP and the correspondent sensitivities on the VF showed a lack of association in healthy subjects whereas 3 out of 4 points were significantly correlated in early glaucomatous patients.

Conclusion: Macular area may present useful morphologic and functional parametersl to distinguish between healthy subjects and early glaucomatous patients. Different parameters show distinct association patterns in each group.

\section{Intravitreal Dexamethasone Implant for Treatment of Persistent Macular Edema in Irvine Gass Syndrome}

\section{S. Mediero, Z. Del Barrio, J. García-Martínez, M. Asencio-Durán, F. Armadá-Maresca}

La Paz University Hospital, Madrid, Spain

Purpose: To evaluate the effectiveness of dexamethasone intravitreal implant $\left(\right.$ Ozurdex $\left.{ }^{\circledR}\right)$ in cystoid chronic macular edema (CME) resulting from Irvine-Gass syndrome.

Methods: Retrospective review to evaluate the effectiveness of a single dexamethasone intravitreal implant over 12 months in 12 eyes with persistent CME resulting from Irvine-Gass syndrome.

Results: Treatment with dexamethasone implant produced a significant improvement in the mean best-corrected visual acuity at month 1 from the baseline mean of 0.17 to $0.27(\mathrm{p}=0.0255)$, and to 
0.27 at month 5 and to $0.29(\mathrm{p}=0.0089)$ by month 12 . The mean Average Central Thickness (ACT) at the baseline was 344.8 and the mean Central Subfield Thickness (CST) was 473.3. A significant improvement in ACT was seen 1 month after treatment with the dexamethasone implant $(294.1, \mathrm{p}=0.0005)$, as well as at $5(312.5$, $\mathrm{p}=0.0048)$ and 12 months $(312.5, \mathrm{p}=0.0496)$. Similarly, a significant improvement in CST was observed 1 month $(303.5, \mathrm{p}=0.0178)$, yet not at 5 months (315.2) and at 12 months after treatment with the implant (337.8). No serious adverse events were detected.

Conclusions: This study suggests that a single dexamethasone implant is a favorable treatment option to improve visual acuity and reduce $\mathrm{CME}$.

\section{Dexamethasone Intravitreal Implant for Treatment of Macular Edema Related to Diabetes and Retinal Vein Occlusion: A 6-Month Follow-Up}

\author{
A. Olate-Pérez, D. Toro-Giraldo, D. Hernández-Pérez, \\ A.M. Duch-Samper \\ Hospital Clínico Universitario de Valencia, Valencia, Spain
}

Purpose: To evaluate safety and efficacy of intravitreal dexamethasone implant $0.7 \mathrm{mg}$ (IDI) in eyes with macular edema secondary to Diabetes (DME) and Retinal Vein Occlusion (RVO) over 6 months.

Methods: Prospective case series study of 21 eyes with DME and 26 eyes with RVO treated with IDI. The outcome measurements were: best-corrected visual acuity (BCVA) and change in central retinal thickness (CRT) by OCT from baseline to week 4, 12 and 24 . Secondary end-point included occurrence of complications.

Results: In the DME group, baseline BCVA was 0.15 (Snellen) and improved to $0.22,0.24$ and 0.23 after 4,12 and 24 weeks respectively. Baseline CRT was $542 \pm 182 \mu \mathrm{m}$ and decreased to $291 \pm$ $94 \mu \mathrm{m}, 278 \pm 182 \mu \mathrm{m}$ and $349 \pm 130 \mu \mathrm{m}$ after 4,12 and 24 weeks. In the RVO group, baseline BCVA was 0.08 (Snellen) and improved to $0.18,0.17$ and 0.12 after 4,12 and 24 weeks respectively. Baseline CRT was $544 \pm 156 \mu \mathrm{m}$ and decreased to $330 \pm 141 \mu \mathrm{m}$, $332 \pm 101 \mu \mathrm{m}$ and $432 \pm 161 \mu \mathrm{m}$ after 4,12 and 24 weeks. The BCVA improvement and the CRT reduction were statistically significant $(\mathrm{p}<0.05)$. Ocular complications were cataracts $(\mathrm{n}=2)$ and ocular hypertension $(\mathrm{n}=3)$.

Conclusion: IDI treatment can improve BCVA and reduce CRT in DME and RVO after 24 weeks. We suggest retreatment after 16 weeks.

\section{Malignant Glaucoma After Cataract Surgery. Role of Neodimium-YAG Laser in the Treatment}

\author{
P. Piñas García, F.J. Hernández Martínez, N. Aznarez López, \\ L. Castillón Torre, A. Trueba Lawand, A. Ruiz Montero, \\ E. Portillo Guerra
}

Hospital San Juan De Dios Del Aljarafe, Sevilla, Spain

Purpose: We show the role of laser treatment in a case of postoperative malignant glaucoma.
Methods: A case clinic report and graphic evolution.

Results: Old woman with cataract N3P3, IOP $14 \mathrm{~mm} \mathrm{Hg}$, gonioscopy grade II, axial length $20 \mathrm{~mm}$, normal retina and optic nerve. 24 hours after phacoemulsification without complications we observed: IOP $55 \mathrm{~mm} \mathrm{Hg}$, corneal edema and athalamia grade III. The treatment included hyperosmotic agents with poor response. Three Nd:YAG iridotomies were not resolutive (pupillary block was discarded); We suspected cilliary block as the etiology of acute glaucoma; a Nd:YAG laser hyaloidotomy and an intensive pharmacological midriasis were completely resolutive.

Conclusion: Malignant glaucoma in a phaco postoperative is a critical clinical situation characterized by athalamia, elevated IOP without retino-choroidal disturbs caused by aqueous misdirection, with anterior displacement of IOL-cilliary diaphragm. We discuss the differents therapies and emphasize the role of laser treatment in this disease.

\section{Predictability of Visual Function Tests in the Alzheimer's Neurodegeneration}

\author{
E.S. García-Martín ${ }^{1}$, B. Rojas ${ }^{1,2}$, R. Hoz ${ }^{1,3}$, Al. Ramírez ${ }^{1,3}$, \\ J.J. Salazar ${ }^{1,3}$, P. Gill'; J.M. Ramírez ${ }^{1,2}$ \\ ${ }^{1}$ Inst Invest Oftalmol Ramón Castroviejo, RETICs OFTALRED \\ RD12-0034-0002, UCM, Madrid, ' ${ }^{2}$ Fac Medicina, UCM, ${ }^{3}$ Fac Óptica \\ y Optometría, UCM, ${ }^{4}$ Unidad Memoria, Servicio Geriatría, Hosp \\ Clínico San Carlos, Madrid, Spain
}

Purpose: Brain atrophy is one of the characteristic signs of Alzheimer's Disease (AD), a neurodegeneration caused by severe loss of neurons in the brain, including several areas known to be involved in visual processing. There are several psychophysical ophthalmological tests that could be used to determine the behaviour of neurons. These tests analyse the actions and cortical processes of retinal ganglion cells. Our purpose was to ascertain the predictability value of some visual tests in mild AD.

Methods: We examined 23 mild AD patients and 27 controls. Patients underwent a complete ophthalmological exam (visual acuity (VA), refraction, contrast sensitivity (CS), colour test, biomicroscopy, IOP and fundoscopy). Inclusion criteria were VA better than 0.6 and no retinal pathology. An aROC analysis was performed to determine the predictability of the different tests.

Results: We found that the tests with the greatest prognostic value in mild $\mathrm{AD}$ patients were $\mathrm{CS}$ for $18 \mathrm{cpd}$ and $12 \mathrm{cpd}$ spatial frequencies, followed by VA, and non-specific errors in the blue region.

Conclusion: We postulate that the psychophysical ophthalmological tests used in this study offer high predictability and could be useful tools for diagnostic support and follow-up in mild AD. 


\section{Choroidal Thickness in Children Measured by Spectral Domain Optical Coherence Tomography}

I. Pinilla Lozano ${ }^{1,3}$, L.X. Herrera ${ }^{1}$, D. Pérez-García ${ }^{1}$ I. Pérez-Navarro', A. Sáchez Cano ${ }^{3,2}$, E. Abecía Martínez ${ }^{4,2}$

${ }^{1}$ Dept. Ophthalmol Hosp. Univ. Lozano Blesa \& School of Medicine, ${ }^{2}$ ISS ARAGON, ${ }^{3}$ Dep Ohthalmol, Hosp Univ Miguel Servet, ${ }^{4}$ Dept. of Applied Physics, Univ of Zaragoza, Spain

Purpose: To evaluate choroidal thickness in children and the repeatability of the technique using Enhanced Depth Imaging Spectral Domain Optical Coherence Tomography (EDI SD-OCT) describing volume differences between all the defined areas of the Early Treatment Diabetic Retinopathy Study (ETDRS).

Methods: Ninety-three eyes of 93 healthy, children were prospectively enrolled. Manual choroidal segmentation on a 25-raster horizontal scan protocol was performed using Spectralis OCT. The measurements of the nine subfields defined by the ETDRS were evaluated.

Results: The studied group was 43 boys and 50 girls with a mean age of $9.62 \pm 2.89$ years. Mean axial length (AL) was $23.19 \pm$ $1.13 \mathrm{~mm}$. Mean subfoveal choroidal thickness (SFCT) was $314.22 \pm$ $55.48 \mu \mathrm{m}$ and mean total choroidal volume was $0.25 \pm 0.04 \mathrm{~mm}^{3}$. Choroidal thickness was higher in hyperopic children (AL, 22.39 \pm $0.83 \mathrm{~mm}$ ); no differences were found between emmetropic (AL, $23.27 \pm 0.54 \mathrm{~mm}$ ) and myopic eyes (AL, $24.33 \pm 0.76 \mathrm{~mm})$. Intra and Inter-observer Intra-class Correlation Coefficient (ICC) showed almost perfect reliability in the measured parameters ranging between $0.929-0.991$ and $0.992-0.998$ respectively.

Conclusion: This study establishes a normal database for choroidal thickness and volume in children. Axial length is associated with choroidal parameters in healthy children with smaller values in hyperopic refraction. EDI-SD-OCT exhibited a high degree of intraobserver and interobserver repeatability.

\section{Vitrectomy Versus Scleral Buckling in Phakic Rhegmatogenous Uncomplicated Retinal Detachment: A Randomized Study}

E. Abecía Martínez ${ }^{1}$, A. Ferreras ${ }^{1}$, I. Pinilla Lozano ${ }^{2}$

${ }^{1}$ Dept. Ophthalmol. Hosp.I Univ., Miguel Servet, ${ }^{2}$ Dept. Ohthalmol. Hosp. Univ. Lozano Blesa \& School of Medicine, Zaragoza, Spain

Purpose: To analyze differences in clinical outcomes between pars plana vitrectomy (PPV) alone, associated to scleral buckle (PPV-SB), an scleral buckling alone (SB) for uncomplicated cases of phakic rhegmatogenous retinal detachment (PRD) in adults.

Methods: One hundred and sixty six consecutive PRD patients were randomized into three groups: VPP $(\mathrm{n}=45)$, VPP-SB $(\mathrm{n}=$ $57)$ and SB $(n=64)$. Exclusion criteria were PVR over Grade B, traumatic or inflammatory history, giant tears, macular hole or impossibility for follow-up. Surgery was carried out by the same surgeon. Groups were homogeneously distributed.

Results: Analysis of pre-operative features did not show differences. No statistical differences were found comparing changes in $\mathrm{BCVA}$ and $\mathrm{PVR}$ incidence $(\mathrm{PPV}=8.8 \%$; $\mathrm{PPV}-\mathrm{SB}=10.5 \%$; $\mathrm{SB}=$
$5.2 \% \mathrm{p}=0.36)$. Differences between primary success were almost significant $(\mathrm{p}=0.056)$ and the SB required more second procedures $(18.7 \%)$; secondary success was $98 \%$. Cataract progression was greater in the PPV and PPV-SB groups.

Conclusion: The study shows no benefits in terms of BCVA improvement or anatomical success between PPV, PPV-SB and SB alone in the repair of PRD. Only cataract surgery has to be performed in a significant higher number of vitrectomy patients.

\section{Oculomotor Palsy After Spinal Puncture}

M. Del-Río-Vellosillo, J.J. García-Medina, M.D. Pinazo-Durán, A. Abengochean Cotaina, F. Acosta-Villegas

Virgen de la Arrixaca University Hospital, Murcia, Spain

Purpose: Oculomotor cranial nerve palsy is a rare, but alarming complication of spinal puncture. A systematic search was undertaken of the literature in english of III, IV and VI cranial nerve palsies after spinal puncture on PubMed.

Methods: The key words used were: 'epidural anaesthesia', 'spinal anaesthesia', 'lumbar puncture', 'dural puncture', 'spinal puncture', 'spinal injection', 'mielography', 'diplopia', 'ophthalmoplegia', 'third nerve palsy', 'fourth nerve palsy', 'sixth nerve palsy', 'oculomotor palsy', 'oculomotor nerve', 'trochlear nerve' and 'abducens nerve'.

Results: Sixty-five articles were identified between 1930 and 2013, and 111 cases were obtained. Subarachnoid anaesthesia was the most frequent cause (46.8\%), with a higher incidence in women. Patient mean age was $39.86 \pm 13.34$ years (range $6-71$ years). VI cranial nerve was the most commonly involved (91.9\%), with higher frequency in the right eye. Palsy onset started $7.19 \pm 3.92$ days after puncture and, in reversible cases, duration was $79.07 \pm$ 81.82 days ( $27 \%$ of the patients recovered within the first month and $17 \%$ did so after more than 6 months). Paresia was permanent in $8.10 \%$ of cases, and only $1.80 \%$ of patients required extraocular muscle surgery.

Conclusion: The horizontal position with analgesics, fluidotherapy and corticosteroids was considered to treat this situation.

\section{Focal Temporal Choroidal Thinning}

R. Dolz-Marco, R. Gallego-Pinazo, M.I. López-Gálvez,

M. Andreu-Fenoll, J.I. Tembl, M. Diaz-Llopis, C. Shields

University and Polytechnic Hospital La Fe, Valencia, Spain

Purpose: To describe the tomographic characteristics of focal temporal scleral bulge with overlying choroidal thinning, a newlyrecognized anatomic variation of macular choroidal thickness (CT) topography.

Methods: Patients undergoing swept-source optical coherence tomography (SS-OCT) examination between October and December 2013 were included. The presence of a focal scleral bulge was evaluated. In those cases with this finding we manually measured the extension of the scleral protuberance and the choroidal shape.

Results: There were 1,044 eyes of 523 patients analyzed and $10 \%$ (99 eyes of 60 patients) showed a focal scleral bulge. The scler- 
al bulge had a mean basal diameter of 3416.11 microns and a mean distance to the foveola of 2183.56 microns. Compared to normal submacular scleral boundary, the scleral bulge was a mean of 110.29 microns higher. Overlying choroidal thickness was 162.94 microns compared to mean submacular thickness of $204.02 \mathrm{mi}-$ crons and uninvolved temporal macular thickness of $276.50 \mathrm{mi}$ crons. The overlying retinal pigment epithelium (RPE) and inner retinal contour were normal in all cases.

Conclusions: On SS-OCT, 10\% of patients show scleral bulge and thinning of the overlying choroid. The clinical significance of this novel sign is unknown.

\section{Reducing the Risk of Developing Diabetic Retinopathy by an Intensive Intervention on Nutrition and Lifestyle}

M.J. Roig-Revert, M. Albert-Fort, F. Santander-Trentini, L. Alonso, J. Marin-Montiel, B. Vivar-Llopis, M.D. Pinazo-Durán

Valencia Study Group in Diabetic Retinopathy (VSGDR), Spain

Purpose: The role of environmental factors in diabetic retinopathy (DR) remains unknown. Dietary and lifestyle measures have health benefits. We evaluated the characteristics of a mediterranean population of diabetics (T1/T2) who were vulnerable to vitreoretinal disorders.

Methods: A multicenter prospective case-control study was carried out in participants of both sexes aged 15-80 years, distributed into three groups: 1$)$ T1DMG $(n=80), 2)$ T2DMG $(n=220)$, and 3 ) controls (CG; $n=100$ ). Half participants of each group were randomly assigned (+OS), or not (-OS) to 1 pill/day of A-O3FA during 18 months of follow-up. Demographics, anthropometrics, personal/familial background, nutrition, life style, ocular examination and blood parameters were recorded and statistically processed by the SPSS 15.0 program.

Results: A high adherence to the Mediterranean Diet was detected. Body mass index (BMI) and familial history of DM, hypertension/cardiovascular disorders were significantly higher in diabetics vs the CG ( $\mathrm{p}<0.001)$. Plasmatic vitamin B12 and folic acid, total antioxidant activity and glutathione were significantly lower, in diabetics $v s$ the CG. These markers ameliorated in the subgroups taking the A-O3FA combination.

Conclusions: We propose that a structured program on dietary and lifestyle modifications leading to weight loss and improve nutritional status should be implemented to reduce the risk of DR.

\section{Lamina Cribrosa Thickness in Axenfeld Rieger Syndrome by Enhanced Depth Imaging}

P. Naranjo Bonilla, R. Giménez Gómez, A.J. Ruiz Alcántara, R. García Catalán, J.M. Gallardo Galera

Hospital Universitario Reina Sofia, Córdoba, Spain

Purpose: To assess Lamina Cribrosa Thickness (LCT) in patients with Axenfeld Rieger Syndrome (ARS).
Case Presentation: Mother and daughter diagnosed with ARS. At the ophthalmic examination they had facial anomalies, posterior embriotoxon (PE) and iridocorneal synechiae, normal intraocular pressure and normal perimetry. The LCT was measured using Spectral Domain Optical Coherence Tomography (SDOCT). The LCT of the mother was $265 \mu \mathrm{m}$ right eye (RE) and 216 $\mu \mathrm{m}$ left eye (LE). The LCT of the daughter was $330 \mu \mathrm{m}$ RE and 388 $\mu \mathrm{m}$ LE.

Discussion: ARS has defects on the synthesis of collagen. According to histological studies PE depends on collagen, in the same way as sclera and LC do. The LCT of the mother was similar to subjects diagnosed with open angle glaucoma. The LCT of the daughter which has 10 years old was similar to control subjects with 55 years old. ARS is usually associated with high levels of IOP and glaucoma. The LC protects against increase of IOP and glaucoma. SD-OCT could be useful in patients with ARS to assess the LC, and their real capacity to tolerate the increase of the IOP.

Conclusion and Home Message: LCT may be thinner in ARS using SD-OCT.

\section{Campimetry of Visual Mechanisms in High Myopics Patients and Its Correlation with Choroidal Thickness}

\author{
M.A. Hernández-Díaz, E. Fernández-López, M. Al Saad, \\ C. Desco-Esteban, M.C. García-Domene, J. Mataix-Boronat, \\ M.I. Luque-Cobija \\ FISABIO Oftalmología Medica (FOM), Valencia, Spain
}

Purpose: To analyze the function of the visual pathways in high myopics patients with an ATD double modulation analyzer and its correlation with choroidal thickness (CT), axial length (AL) and best corrected visual acuity (BCVA).

Methods: We conducted a prospective observational pilot study. We included 12 eyes from 8 high myopic patients. We performed four campimetries by ATD double modulation analyzer using four sinusoids with different spatio-temporal frequencies: two achromatic mechanisms to study the magnocellular and parvocellular achromatic pathways, red-green to analyze the parvocellular chromatic pathway and blue-yellow for the koniocelular pathway. The AL was calculated using IOL-Master biometry and the subfoveal CT was measured with Spectralis OCT by Heidelberg.

Results: The age of the patients was $49 \pm 9$ years. The mean refractive error was $10 \pm 4 \mathrm{D}$ and the mean BCVA was $0.9 \pm 0.4$. The average of AL was $28 \pm 2 \mathrm{~mm}$ and the mean of CT was $125 \pm 85 \mu \mathrm{m}$. We found a correlation between the BCVA and visual field defects, but there were no correlation with AL and CT.

Conclusion: When analyzing the loss of visual sensitivity in the visual pathways of high myopics patients we have observed that chromatic mechanisms are the first to be affected, correlating such losses with visual acuity. 


\section{Episcleral Macular Buckling for Macular Hole in Highly Myopic Eyes. Matching the Fovea is Really Necessary?}

\author{
R. Araújo Miranda Villaverde Lopes, E. Fernandez Lopez, \\ J. Mataix Boronar, E. Palacios Pozo, A. Navea Tejerina
}

Fisabio Oftalmologia Médica (FOM), Valencia, Spain

Purpose: To evaluate the importance of the episcleral implant location in the anatomical and visual outcomes of macular hole in highly myopic eyes with posterior staphyloma.

Methods: This retrospective interventional case series includes 9 eyes of 9 high myopic patients with macular hole and foveal detachment or retinoschisis with posterior staphyloma. Pars plana vitrectomy combined with NPB macular buckling (AJL ophthalmic) was performed in all patients. Best corrected visual acuity (BCVA), optical coherence tomography and optical biometry to measure axial length were performed in all cases before and after surgery.

Results: Macular hole closing rate was $100 \%$, mean BCVA improved in 7 eyes $(77.8 \%)$ after surgery at the end of the follow-up. The remaining 2 eyes did not recovery useful visual acuity, one of the cases had a long-standing retinal detachment before surgery, other suffered an ischemic optic neuropathy. In spite of the $100 \%$ rate closure of the macular hole, just 2 of the 9 explants were perfectly centered on the fovea.

Conclusion: Combined pars plana vitrectomy and macular buckling is an effective technique to treat myopic foveoschisis and macular hole with both anatomical and visual improvement. Despite some explants were not exactly subfoveal we have observed favorable clinical outcomes.

\section{'En Face' Swept-Source Optical Coherence Tomography Features of Polypoidal Choroidal Vasculopathy}

R. Arranz, C. Marín-Lambies, R. Fons, J. Marin-Montiel, C. Arroyo, F. Marín

Hospital Clínico Universitario Valencia, Spain

Purpose: To report 'en face' swept source optical coherence tomography (SS-OCT) findings in patients with choroidal polypoidal choroidal vasculopathy.

Methods: We have studied a series of patients with polypoidal choroidopathy by SS-OCT (Topcon DRI Atlantis). In all of them an FA and ICG study was conducted and we have compared the characteristics of both examinations to assess the clinical utility of 'en face' exploration.

Results: Choroidal polyps can be relatively difficult to detect with FA and ICG in some cases. In our results, 'en face' SS-OCT provides more detailed visualization of polyps and their differentiation from the accompanying changes in the fundus.

Conclusion: 'En face' study with SS-OCT appears as a new additional valuable tool in the diagnosis of PVC and its associated disorders.

\section{The 'Pitchfork Sign' in Young Adults with Choroidal Neovascularization}

C. Arroyo, C. Marín-Lambies, R. Fons, F. García-lbor, J. Sifre, F. Marín

Hospital Arnau de Vilanova, Valencia, Spain

Purpose: The purpose of this study was to assess the presence in young patients with choroidal neovascularization (CNV) of a new optical coherence tomography sign that has been reported as characteristic of inflammatory CNV.

Methods: We retrospectively reviewed twelve eyes of twelve patients with choroidal neovascularization not associated with age related macular degeneration, pathologic myopia, angioid streaks or hereditary diseases. We evaluated the presence of pitchfork sign in the first OCT study and its evolution during treatment with anti VEGF.

Results: In seven cases we found the pitchfork sign of different intensity. In three of them we can not find other associated abnormalities in the affected or the contralateral eye. The disappearance of this sign is rapid after treatment with anti-VEGF in all cases.

Discussion: The pitchfork sign has been described as a marker of inflammatory CNV and is relatively common in young adults with this type of NVC, but we have found that this sign is also present in cases considered as 'idiopathic' CNV. A longer follow-up of these eyes and larger series is necessary to rule out possible inflammatory etiology of these neovascular membranes.

\section{Biomarkers and Surrogate Clinical Endpoints for the Development and Progression of Diabetic Retinopathy in Patients with Type Two Diabetes Mellitus}

\section{B. Vivar-Ilopis, F. Santander-Trentini, M.J. Roig-Revert, C. Galbis-Estrada, L. Alonso, M. Albert-Fort, M.D. Pinazo-Durán}

Unidad de Investigación Oftalmológica 'Santiago Grisolía', Valencia, Spain

Purpose: Identify potential risk factors and biomarkers of diabetic retinopathy in type 2 diabetes in a Mediterranean population to prevent vision loss.

Methods: We propose a multicenter case-control study which involved 10 centers in the Valencian community. 360 patients, distributed in 2 groups: DM2 and healthy controls were selected. All of them were made a Personal interviews with family, anthropometric, nutritional data, eye examination (AVMC; Biomicroscopy; PIO; fundus; Retinography; OCT) Total and classical biochemical determinations, glutathione, MDA and antioxidant (AOXT).

Results: Glycosylated hemoglobin, homocysteine, C-reactive protein, cholesterol, triglycerides, folic acid, MDA and AOXT proved to be a significant predictor of $\mathrm{RD}(\mathrm{P}<0.05)$. The history of DM and hypertension, the Mediterranean diet adherence and duration of disease $(\mathrm{P}<0.05)$ also proved to be a major risk factor.

Conclusion: In addition to strict glycemic control and ophthalmic examination it is important to control these factors that can 
be modified in order to prevent visual loss. We suggest to use blood analysis to identify potential biomarkers that may help those patients vulnerable to RD.

\section{Eighteen Months of Follow Up in the Valencian Study on Diabetic Retinopathy}

F. Santander-Trentini, B. Vivar-llopis, M.J. Roig-Revert,

C. Galbis-Estrada, L. Alonso, M. Albert-Fort, M.D. Pinazo-Durán

Ophthalmic Research Unit 'Santiago Grisolía', Valencia, Spain

Purpose: A multicenter study on the Valencian Community population was designed to identify patients with early signs of diabetic retinopathy (DR) but not symptoms, to integrate data that allow providing effective biomarkers to obtain an overall reduction of DR progression.

Methods: From baseline participants were distributed into type 1 and 2 diabetics (T1D; T2D) and healthy controls (CG). One pill/day of combined antioxidants and omega 3 fatty acids (A/ $/ 23)$ was randomly administered to half participants in each group. At 18 months of follow-up the remaining 246 patients were evaluated. Personal interview, best corrected visual acuity, retinographs and ocular coherence tomography examination were done. Plasma samples were collected and processed to determine classical molecules and those involved in oxidative stress, vascular/neurological risk. Statistical analysis was performed by the SPSS 15.0 program.

Results: Significant biochemical changes were observed between the T1D-T2D with the A/ $\omega 3$ respect those without A/ $\omega 3$ and the CG $(p<0.05 ; p>0.01 ; p<0.001)$. Main alterations were detected in the levels of homocysteine, folic acid, vitamin B12, malondialdehyde, total antioxidant activity and gluthatione.

Conclusions: We are currently perform this work that will last for three years, for assessing the use of $\mathrm{A} / \omega 3$ in diabetics, results of which have to be awaited.

\section{Correlations Between Corvis ST Parameters, IOP and CCT}

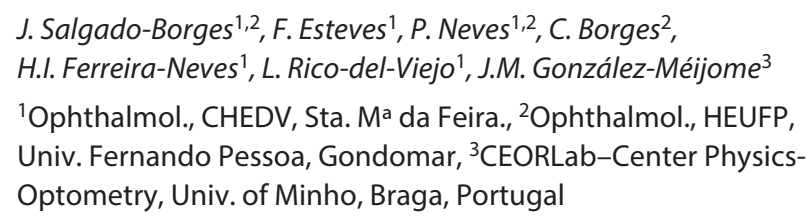

Purpose: To evaluate correlations of biomechanical parameters from Corvis ST, intra-ocular pressure (IOP) and central corneal thickness (CCT) in healthy individuals.

Methods: 86 eyes (58F/18M; $44 \pm 8$ years) were evaluated in the morning with Corvis ST including biomechanical parameters related to first applanation (A1), second applanation (A2) and highest concavity (HC). Comparisons were performed by independent sample T-test. Kolmogorov-Smirnov test evaluated normality distribution and non-parametric Spearman's Rho correlation coefficient $(\mathrm{R})$ was used.
Results: IOP and CCT were poorly correlated $(\mathrm{R}=0.304 ; \mathrm{p}=$ 0.004). A1 parameters were correlated with IOP: high for A1Time $(\mathrm{R}=0.984 ; \mathrm{p}<0.001)$, inverse and moderate for A1Velocity $(\mathrm{R}=$ $0.453 ; \mathrm{p}<0.001)$ and low for A1Length $(\mathrm{R}=0.253 ; \mathrm{p}=0.019)$. Only two A2 parameters were correlated with IOP: high and inverse for A2Time $(\mathrm{R}=-0.810 ; \mathrm{p}<0.001)$, and moderate for A2Velocity $(\mathrm{R}=$ 0.649 ; $\mathrm{p}<0.001)$. For Highest concavity, there was an inverse correlation between IOP and DefAmpMax and PeakDistance $(\mathrm{R}=$ -0.764 and $\mathrm{R}=-0.626 ; \mathrm{p}<0.001)$ and a direct correlation with HCRadius $(\mathrm{R}=0.488 ; \mathrm{p}<0.001)$. Correlations of CCT with A1Time, A2Velocity, PeakDistance and HCRadius were significant $(\mathrm{p}<0.006)$ but weak $(\mathrm{R}<0.450)$.

Discussion: Several parameters from Corvis ST are correlated with the IOP. A1Length and A2Length as well as HCTime and HCRadius are the parameters less correlated with IOP. Correlations of Corvis ST parameters with CCT are generally poor.

Conclusion: Corvis may clarify the behavior of cornea in glaucoma patients.

\section{Accommodation Amplitude and Visual Acuity of the Accommodative Intraocular Lens, the Akkolens Lumina}

\section{J. Alió, A. Angelov, A. Simonov, M. Rombach}

Vissum Corporation, Alicante, Spain

Purpose: To evaluate the accommodation and the visual rehabilitation provided of the new Akkolens Lumina accommodative IOL (AIOL).

Methods: 42 eyes were included in this study. 2 groups of eyes were differentiated according to the IOL implanted. Group A, 31 eyes implanted with the AIOL Akkolens Lumina and Group B, 11 eyes implanted with the monofocal IOL Acrysof SA60. Visual acuity, defocus curve, contrast sensitivity and the objective accommodation with the WAM autorefractometer were measured at 12 months.

Results: Significant better uncorrected near (UNVA) and distance corrected near visual acuities (DCNVA) were observed for group A ( $p \leq 0.01)$. Better visual acuities were found in the defocus curve for group A for defocus levels from -4.0 to $-0.5 \mathrm{D}(\mathrm{p} \leq 0.02)$. Better visual acuities for the AIOL were observed in the depth of focus studied by defocus curves for visual acuities of $0.8,0.6$ and 0.4 ( $\mathrm{p} \leq 0.02$ ). No statistical differences were detected between groups in any spatial frequency of the mesopic contrast sensitivity $(\mathrm{p} \geq 0.28)$.

Conclusion: The Akkolens Lumina successfully restores the visual function with good near visual acuity due to the accommodation provided. Contrast sensitivity is similar to a monofocal IOL. 


\section{Keratopigmentation in a Keratoconus Patient after Keratoplasty}

J.L. Alió, A.E. Rodríguez, M. El-Bahrawy

Vissum Corporation, Alicante, Spain

Purpose: To describe the successful use of a double keratopigmentation technique (Femtosecond assisted and Superficial) to manage a corneal opacity as consequence of a trauma following a keratoplasty.

Case Presentation: A 45 year old female patient was diagnosed with bilateral keratoconus and underwent keratoplasty in both eyes with good visual outcomes of 0.7 and 0.46 for right and left eyes, respectively. One year after the surgery, she had a tennis ball trauma to the left eye, causing retinal detachment for which she underwent vitrectomy in this eye with resuturing of the corneal graft. On examination left eye presented poor cosmetic appearance due to extensive scarring of the corneal graft with no perception of light. To restore the cosmetic appearance a keratopigmentation was done using both Femtosecond assisted and Superficial technique to simulate the iris. Micronized brown pigment (VEMP204) was used.

Discussion: A second surgical enhancement was done using a darker brown pigment (VEMP200). After one year of follow up, the patient expressed satisfaction with the excellent cosmetic appearance.

Conclusion and Home Message: Femtosecond assisted keratopigmentation combined with the Superficial technique were used to achieve a pigmented replica of the iris on the cornea restoring the cosmetic appearance of the eye.

\section{Acute Onset of Bilateral Papilledema after Cessation of Oral Topiromate}

\author{
J. Fares-Valdivia, J.J. García-Medina, M. Del-Río-Vellosillo, \\ S. Valentino, M.B. Rodríguez-Cavas, M. Tudela-Molino, \\ C. Gómez-Molina
}

Hospital General Universitario Reina Sofía, Murcia, Spain

Purpose: Topiromate (TPM) is a drug used in the treatment of epilepsy, migraine and benign intracranial hypertension (BIH). This case describes an exceptional side effect after the cessation of this medication.

Case Presentation: A 20 year-old woman who attended the emergency department complained about acute blurred vision with both eyes. She had been treated with TPM for 2 weeks because of migraine without aura. Visual acuities (VAs) were 0.05 in the right eye (RE) and 0.1 in the left eye (LE). Autorrefraction revealed $-7.50-2.00 \times 85^{\circ} \mathrm{RE}$ and $-7.75-1.00 \times 90^{\circ} \mathrm{LE}$. VAs with these corrections achieved 1 in both eyes. Biomicroscopy and fundoscopy were normal in both eyes. Intraocular pressure (IOP) was $26 \mathrm{~mm} \mathrm{Hg} \mathrm{RE}$ and $20 \mathrm{~mm} \mathrm{Hg} \mathrm{LE}$. Acute myopia and ocular hypertension secondary to TPM were suspected so the cessation of this drug was advised. One week later the refraction, VAs and IOP became normal. However fundoscopy revealed an asymmetric bilateral papilledema that was confirmed by means of OCT. The patient was derived to the department of neurology with the suspicion of BIH. This diagnosis was confirmed after computerized tomography and lumbar puncture with laboratory tests.

Conclusion and Home Message: Cessation of TPM may be related to acute onset of bilateral papilledema when $\mathrm{BIH}$ is present.

\section{Spontaneous Corneal Clearing After latrogenic Descemetorhexis of the Superior Hemicornea with Involvement of the Visual Axis: Eight-Month Follow-Up \\ M. Tudela-Molino, J.J. García-Medina, M. Alcaraz-Albaladejo, \\ D. Sánchez-Martínez, M.B. Rodríguez-Cavas, J. Fares-Valdivia, \\ R. Gutiérrez-Ortega \\ Hospital General Universitario Reina Sofía, Murcia, Spain}

Purpose: Until recently it has been considered that the endothelium had a very limited capacity of proliferation but there are cases, like this one, that indicate otherwise.

Case Presentation: Patient who suffered unnoticed descemetorhexis of superior hemicornea in the left eye during a cataract surgery without any other complication. The day after, the patient showed a severe edema of superior hemicornea. The best corrected visual acuity (BCVA) after 1 and 2 months was 0.3 and 0.4 repectively. However three months after the operation, the edema showed a significant improvement and the BCVA was 0.9 and 8 months later it was 1 . At this moment the central endothelial cell counting was 925 cells $/ \mathrm{mm}^{2}$ with a transitional area between cellular and acellular endothelium in the superior hemicornea. The case is illustrated with slit-lamp photographs, Pentacam maps (paquimetric and Scheimpflug maps) and confocal microscopy.

Conclusion and Home Message: A spontaneous improvement of corneal transparency, paquimetry and visual acuity can take place about 3 months postoperatively after a iatrogenic descemetorhexis in superior hemicornea with involvement of visual axis. This change may be due to endothelial cell proliferation over 'naked' corneal stroma (without Descemet) and it is maintained over the time.

\section{X-Chromosome Linked Juvenile Foveal Retinoschisis \\ I. Pinilla-Lozano1,3, L.X. Herrera' , D. Pérez-García1 , J. Ibáñez', C. Peiró ${ }^{1}$, E. Abecia-Martínez ${ }^{2,3}$ \\ ${ }^{1}$ Dep Ophthalmol. Hosp Univ. Lozano Blesa and School of Medicine, ${ }^{2}$ ISS ARAGON, ${ }^{3}$ Dep Ohthalmol. Hosp Univ. Miguel Servet, ${ }^{4}$ Dep Applied Physics, University of Zaragoza, Spain}

Purpose: Juvenil familiar retinoschisis is an inherited retinal disorder associated to chromosome $\mathrm{X}$. Two cases are presented and optical coherence findings are described.

Methods: Two young males, aged 26 and 24 years affected by macular retinosquisis are presented. Any of them had other family members affected.

Results: Both males showed the present of macular retinoschisis with peripheral quisis at the inferior temporal retina. Visual acuity of case 1 was $2 / 10$ at both eyes and the second one 5/10 and 
$1 / 10$ in right and left eye respectively. An optical coherence tomography study was performed and the findings showing cysts in both nuclear layers persistence of few Henle fibers and a diminish thickness of the Internal Limiting Membrane. Both of them showed high foveal thickness.

Conclusion and Home Message: Juvenil familiar retinoschisis is an uncommon inherited retinal disorder Differential diagnosis is discussed, as well as the role and findings of optical coherence tomography for the diagnoses.

\section{Acute Onset of Facial Nerve Paralysis After Cataract Surgery Under Peribulbar Anesthesia}

J.J. García-Medina, M. Del-Rio-Vellosillo, M.B. Rodríguez-Cavas,

M. Tudela-Molino, J. Fares-Valdivia, A. Guardiola-Fernández, M.D. Pinazo-Durán

Hospital General Universitario Reina Sofía, Murcia, Spain

Purpose: To describe an exceptional complication after cataract surgery under peribulbar anesthesia.

Case Presentation: A 43 year-old diabetic man underwent cataract surgery of the right eye under peribulbar anesthesia. When the eye was unpatched the day after the patient presented a typical case of right peripheral facial nerve paralysis that was not present the day before. He complained of cephalea since he was operated. The patient did not complained about any other neurological symptoms. He was sent to the department of neurology for assessment that did not add further findings. Laboratory tests, cranial computerized tomography, cerebral magnetic resonance, chest radiograph, echo-doppler of supra-aortic vessels did not show any pathological data. After six-month followup the signs and symptoms disappeared progressively.

Conclusion and Home Message: Cataract surgery under peribulbar anesthesia may be associated to the acute onset of ipsilateral peripheral facial nerve palsy. This entity may be benign and self-limited after some months.

\section{Key Findings in Choroidal Osteoma Diagnose: Importance of the Imaging Tests}

\section{Sánchez Martínez, I. Salido Salido, M. Tudela Molino,}

\section{Selles Navarro}

Hospital General Universitario Reina Sofía, Murcia, Spain

Purpose: Due to low incidence of choroidal osteoma, we consider to do an update for its differencial diagnostic with new tecnics of imagen like' Enhance Depth Imaging Optical Coherence tomography (EDI-OCT)'.

Case Presentation: 65-years-old diabetic patient, with diagnosed and treated prostate cancer. BCVA was 20/20 in both eyes. Fundoscopy revealed a $5 \mathrm{~mm}$ wide geographical area in OS, bloodsurrounded, next to the temporal superior venous branch. OCT revealed a choroidal mass with cage-like reflective pattern. B-Scan showed a calcic-appearance lesion.
Discussion: Suspecting choroidal metastasis, patient was examined by internal medicine without positive results. The fluorescein and indocianine angiography was performed and results were not suggestive of hemangioma or melanoma. The EDI-OCT imagen contributes sensible information that suggest a choroidal osteoma. Two months later BCVA of OS is 20/40 due to choroidal neovascularization. Intravitreal injection of Ranibizumab improved BCVA but failed to reduce of choroidal mass.

Conclusion and Home Message: Choroidal osteoma is a benign tumor in which bone replaces the choroid. Differencial diagnose must be done, specially using imaging tests. OCT revealed the cage-like pattern, while B-scan pointed out the calcic nature of this lesion and indocianine test could differenciate between osteoma and hemangioma. These were the key findings that allowed us to identify this unusual tumor.

\section{Macular Hole after Ranibizumab Treatment in a Case of Central Retinal Vein Occlusion and Resolution with Dexamethasone Implant}

\author{
E. Abecia Martínez ${ }^{1}$, P. Calvo ${ }^{1}$, I. Pinilla Lozano ${ }^{2}$ \\ ${ }^{1}$ Dep Ophthalmol Hosp Univ Miguel Servet, ${ }^{2}$ Dep Ohthalmol \\ Hosp Univ Lozano Blesa and School of Medicine, Zaragoza, \\ Spain
}

Purpose: To present a case of macular hole developed in a CRVO and resolved after dexametasone implant.

Methods: We describe a case of a macular hole development after intravitreal ranibizumab injection and its posterior closure after dexamethasone implant in a patient with CRVO. A 59 yearold man was referred to our department for a long term visual impairment in his left eye. Best corrected visual acuity (BCVA) was $20 / 25$ in the right eye (RE) and 20/400 in the left eye (LE). Binocular ophthalmic fundus examination revealed signs compatible with the CRVO diagnosis. Spectral-domain optical coherence tomography (SD-OCT) confirmed macular edema (central retinal thickness was $794 \mu \mathrm{m}$ ) and neurosensory detachment.

Results: Intravitreal injection of ranibizumab was indicated and carried out without any intraoperative incidence. At the first week follow-up examination SD-OCTshowed the resolution of macular edema and the presence of a full-thickness macular hole. After 1-month follow-up, the ME increased and a partial closure of the hole was observed. Dexamethasone intravitreal implant was then indicated. In the subsequent 1 month of follow-up ME resolved, macular hole closed and BCVA was 20/120.

Conclusion: Our report describes the evolution of a complication associated with ranibizumab intravitreal injection and its resolution after dexamethasone intravitreal implant. 


\section{Postoperative Endophthalmitis in Retinal Detachment: Clinical Case}

\begin{abstract}
P. Piñas-García, F.J. Hernández-Martínez, L. Castillón-Torre, M.E. Tena Sempere, M.E. Mantrana-Bermejo, S. López-Aramburu

Hospital San Juan de Dios del Aljarafe, Sevilla, Spain
\end{abstract}

Purpose: Highlight the importance of early diagnosis and management of this serious postoperative complication.

Case Presentation: Varon 50 years old, without systemic disease. He went to emergency with painless of monocular vision since a month ago. He had a total retinal detachment and was operated with cerclage and pars plana vitrectomy without incident. At 72 hours, he debuted with a very aggressive postsurgical endhopthalmitis. We perfomed a vitrectomy of emergency with take sample and injection of intravitreal antibiotics. We had got to control the infection and the retina applied although the patient presented a dramatic fundoscopy image of Neurorretinitis.

Discussion: The infectious endophthalmitis is one of the most serious complications in clinical practice of the ophthalmologist. In $90 \%$ of cases is due to cataract surgery. We present our experience in handling a case of endhopthalmitis after a surgical retinal detachment.

Conclusion and Home Message: The incidence of this disease is very low but it is key to make an early clinical diagnosis to act immediately and get resolution of infection intraocular with the least damage.

\section{Naxos Disease and Carvajal Syndrome. Management of Ophthalmologic Manifestations}

\section{A.J. Ruiz-Alcántara, R. Giménez-Gómez, P. Naranjo-Bonilla, R. García-Catalán, J.M. Gallardo-Galera \\ Ophthalmology Department, Reina Sofía University Hospital, Córdoba, Spain}

Purpose: To assess the effectiveness of tacrolimus in treating severe dry eye syndrome in a case of Naxos disease.

Case Presentation: A 7-year-old male diagnosed with Naxos disease was transferred to our Center for assessment of severe dry eye. Parents were concern about intense photophobia, watery eyes and red eye. Visual acuity was 0.1 in both eyes. Bilateral lower lid mild ectropion, conjunctival hyperemia with signs of squamous metaplasia and paracentral leucoma were shown in biomicroscopy. Tear meniscus was lower than $0.4 \mathrm{~mm}$ and BUT was 1-2 seconds. Fundus examination was normal. After several treatments, the patient was treated with Tacrolimus $0.03 \%$ ophthalmic ointment twice a day. After 8 months, symptoms improved, tear meniscus increased and visual acuity improved to 0.5-0.6.

Discussion: Inflammation is the main mechanism in the perpetuation of dry eye. Therefore, tacrolimus, by decreasing the degree of T-cell mediated inflammation, may control the manifestations and symptoms of dry eye.

Conclusion: Topical treatment with tacrolimus $0.03 \%$ may be an alternative in cases of severe dry eye that have not responded to standard treatments. Further studies are necessary to assess the long-term efficacy and safety.

\section{Uveal Melanoma: Conservative Surgery without Brachytherapy}

\author{
A. Fidalgo-Broncano, E.J. Infantes Molina, A. Arias-Palomero, \\ L.B. Alfaya Muñoz, M.J. Domínguez Fernández, \\ M. Pradas-González, F. González-del-Valle
}

Mancha Centro Hospital, Alcázar de San Juan, Spain

Purpose: To show our surgical approach for uveal melanoma resection preservating ocular function/anatomy.

Description: We perform a radical excision of the tumor leaving a wide security margin associated to a $23-25 \mathrm{G}$ pars plana vitrectomy. Intraoperative delimitation of the tumor size and the security margins are assisted by an endolight. The tumor is excised with sclera and retina. A large scleral flap allow us to cover residual defects after removing the tumor. If necessary we perform scleral cryotherapy, cataract surgery, scleral buckle, cyclectomy or iridectomy. If the retina is excised silicone oil is introduced. Systemic hypotension did not appear in any cases.

Discussion: Best current treatment for uveal melanoma is discussed. Last conclusions from 'the Collaborative Melanoma Study Group' indicates that there are no differences between brachytherapy/enucleation in terms of survival. Brachytherapy associated severe toxic complications (radioactive retinitis) excluding our main goal of properly studying the cellular lineage of the tumor. Systemic hypotension could be an additional complication in this patient.

Conclusion and Home Message: Conservative surgery allows us to histologically analyze the melanoma and to preserve the ocular anatomy and function without the complications of the brachytherapy.

\section{Enophthalmos as the First Manifestation of Non Diagnosed Breast Cancer}

\section{F. Santander-Trentini, A. Solves-Alemán, L. Domingos-Gid, M. Rosell-Domínguez}

Hospital Dr. Peset, Valencia, Spain

Purpose: Diagnose breast cancer through an isolated enophthalmos case.

Case Presentation: A seventy-seven-year-old woman who presented a 3 months binocular diplopia, associated with a $12 \mathrm{~mm}$ unilateral enophthalmos with pseudoptosis, strabismus, and restriction of extraocular movement.

The orbital MRI and CT scan showed a density alteration of the intraconal fat of the right eye, with tissue that compromised both rectal medius and inferior muscles, adjacent to the optic nerve, producing enophthalmos. Suspecting a possible metastasis, a breast ultrasound scan and a thoracic-abdominal-pelvic TC scan was performed, finding a bilateral breast tumor with right axillary adenopathies. Breast and extraocular muscle biopsy resulted in an infiltrating lobular carcinoma.

Discussion: There are multiple existing causes of enophthalmos, including different types of cancer, post-traumatic enophthalmos, among other etiologies. Enophthalmos associated to breast cáncer must be considered in female patients with no past 
history of traumatism and at age of risk. Tends to be chronic or sub-acute, corresponding to a contractile metastasis with no inflammatory signs. It could present itself as an initial sign allowing breast cancer diagnosis in previously asymptomatic patients.

Conclusion and Home Message: Enophthalmos could be the first manifestation of breast cancer and must be taken into account in affected patients with unknown etiology.

\section{Diagnosis of Cone Dystrophy with Optical Coherence Tomography (OCT)}

\author{
R. De Hoz ${ }^{1,2}$, E.S. García-Martín ${ }^{1}$, B. Rojas ${ }^{1,3}$, A. Triviño ${ }^{1,3}$, \\ J.M. Ramírez ${ }^{1,3}$ \\ ${ }^{1}$ Inst Invest Oftalmol Ramón Castroviejo, ${ }^{2} \mathrm{Fac}$. Óptica- \\ Optometria, ${ }^{3}$ Fac. Medicina, UCM, Madrid, Spain
}

Introduction: Macular cone dystrophy is an inherited bilateral disorder whose incidence is $1 / 400.000$. It usually begins between the first and the third decade of life. Optical Coherence Tomography (OCT) is a well-established method of examining the retinal architecture in real time in situ.

Case Presentation: A 28-years-old woman came complaining of progressive loss of visual acuity and photophobia since childhood. Her vision was better at night and had no color discrimination. Her best corrected visual acuity was 20/30 in both eyes. Fundoscopic examination revealed a pale papilla and vascular narrowing, no pigment in the macula and diffuse alteration of the RPE OU. Automated perimetry revealed a diffuse reduction in sensitivity and a macular scotoma in both eyes. Farnsworth showed an unspecific color alteration. The macular OCT scan images showed a clearly boundared area underneath the fovea of loss of the highly backscattering photoreceptor layer that could correspond with macular cone atrophy in both eyes.

Conclusions: The OCT allows us to know the retina tissue structure with high resolution in vivo. In this degenerative disease permit us to observe a low intraretinal reflectivity in the macular area that is the virtual space who are in the area who leaves the outer photoreceptor segments that never developed.

\section{AntiVEGF Treatment for Juxtapapillary Capillary Hemangioma \\ M.R. Fons-Martínez ${ }^{1}$, C. Marín-Lambíes ${ }^{2}$, E. Tarragó-Simón ${ }^{1}$ \\ ${ }^{1}$ Hospital Lluís Alcanyís, Xàtiva, ${ }^{2}$ Hospital de Manises, Valencia, Spain}

Purpose: To present results of the treatment with intravitreal (iv) antiVEGF in 2 cases of juxtapapillary retinal capillary haemangioma ( $\mathrm{RCH})$

Methods:

Case 1: A 72-year old man with a juxtapapillary RCH and lipid exudation extending into the macula was treated with two monthly iv injections of $125 \mathrm{mg}$ bevacizumab as the sole treatment.

Case 2: A 67-year old man, with bilateral diabetic macular edema, who presented juxtapapillary $\mathrm{RCH}$ without exudation in his right eye, was treated with just one injection of ranibizumab.
In both cases best corrected visual acuity (BCVA), fundus fluorescein angiography (FFA) and optical coherence tomography (OCT) were performed at baseline and after treatment with a follow-up of 6 years in case 1 and 1 year in case 2 .

Results: After treatment in case 1 a complete regression of lipid exudates and subretinal fluid was observed. In case 2 the tumor completely disappeared.

Conclusions: Our cases underline that both antiVEGF (bevacizumab or ranibizumab) treatment as a monotherapy can be very useful in the juxtapapillary RCH.

\section{Fundus Albipunctatus vs Retinitis Punctata Albescens. A Case Report \\ A. González-Gómez, J.M. Sandoval-Codoni, A. Soler-García, M. González Gómez, M.J. Morillo Sánchez}

HCU Virgen de la Victoria, Málaga, Spain

Purpose: Emphasize the importance of correct diagnosis of fundus flecked lesions.

Case Presentation: A 25 year old man who manifested nyctalopia. He had been given a diagnosis of Retinitis pigmentosa (RP). The best corrected visual acuity was unity in both eyes. Perimetry SITA 30-2 was within normal limits. Symmetric small yellowwhite flecks were observed from the midperiphery, without affecting the macular area. There were no changes in papilla or vessels. The ERG showed an abnormal response at all stages with normalization after a period of dark adaptation. So we confirmed the diagnosis of Fundus albipunctatus (FA).

Discussion: FA is an inherited disease characterized by nyctalopia. These patients maintain a good visual acuity and a normal visual field. However, RP albescens manifests a progressive visual acuity and visual field loss. There is a retinal vascular attenuation and papillary pallor too. Furthermore, the scotopic ERG waveforms usually do not regenerate.

Conclusions: It is essential to differentiate between these two entities because visual prognosis in FA is not serious enough to prevent from leading a relatively normal life. Ensuring an accurate diagnosis is important for the emotional state of our patients because visual prognosis is different.

\section{Is Optical Coherence Tomography (OCT) Useful to Find Differences Between Subtypes of Multiple Sclerosis?}
A. Soler-García, A. González-Gómez, J.M. Sandoval-Codoni
Hospital Cínico Universitario Virgen de la Victoria, Málaga, Spain

Purpose: To evaluate differences in thickness of nerve fiber layer (RNFL) between patients with Multiple Sclerosis (MS), according to clinical subtype of disease: Secondary progressive (SPMS) or relapsing-remitting (RRMS).

Methods: A cross sectional study in 61 patients affected by MS classified into 2 groups: RRMS or SPMS. Best corrected visual acu- 
ity (BCVA), intraocular pressure (IOP) and OCT to quantify the thickness of the nerve fiber layer were obtained.

Results: Average thickness of RNFL (RNFLav) in patients with RRMS: $94.64 \pm 16.74 \mu \mathrm{m}$ with respect to patients with SPMS: $93.77 \pm 11.62 \mu$. Analysis by sectors of the RNFL showed the following results in subtype RRMS: temporal sector (RNFLt) $61.44 \pm 15.7 \mu \mathrm{m}$, nasal sector (RNFLn) $83.35 \pm 24.24 \mu \mathrm{m}$, superior sector (RNFLs) $118.80 \pm 23.9 \mu \mathrm{m}$ and inferior sector (RNFLi) $115.58 \pm 22.7 \mu \mathrm{m}$ respectively. In SPMS subtype we observed these results by quadrants: RNFLt $59.57 \pm 15.67 \mu \mathrm{m}$, RNFLn $81.64 \pm 16.4 \mu \mathrm{m}$, RNFLs $115.07 \pm 15.6 \mu \mathrm{m}$ and RNFLi $115.07 \pm$ $17.4 \mu \mathrm{m}$ respectively.

Conclusions: Analysis of the thickness of the nerve fiber layer showed no significant differences between subtypes of MS.

\section{Reduced Retinal Nerve Fiber Layer Thickness in Children with Severe Obesity}

\author{
J. Pacheco Cervera, P. Codoñer-Franch, R. Simó-Jordá, \\ S. Pons-Vázquez, C. Galbis-Estrada, M.D. Pinazo-Durán \\ Hospital U. Dr. Peset, Valencia, Spain
}

Purpose: Childhood obesity is increasing in recent years with a significant impact on health. Current studies on obese children have not established yet a relationship with its effects on the eyes and vision. We analyze the consequences of obesity in the optic nerve outcomes measuring retinal nerve fiber layer (RNFL) by optical coherence tomography imaging.

Case Presentation: We performed a prospective cross-sectional, observational study in ninety-seven children, which were distributed according to body mass index. Anthropometric measurements, blood determinations of parameters of metabolic risk and leptin, adiponectin and interleukin-6 were measured. Also ophthalmologic complete examination and retinograps on the retina and optic nerve and optical coherence tomography of the head of the nerve with Cirrus OCT Zeiss, 'Optic Disc Cube 200x200.

Discussion: It was observed a statistically significant global decrease of the RNFL thickness with the severe increase of body mass index. A significant inverse correlation of adiposity indices with RFNL thickness was found.

Conclusion and Home Message: These findings suggest that adiposity and the secondary inflammatory state is a linked factor to diminished nerve optic fibers, and highlights the relevance of eye care follow-up in severe obese children in order to prevent visual impairment and blindness.

\section{Peripapillary Lesion: Tumoral or Inflammatory? Case Report}

J.M. Sandoval Codoni, A. Soler García, A. González Gómez

HCU Virgen de la Victoria, Málaga, Spain

Purpose: Report a case of a pigmented peripapillary lesion in a young subject which require to make a broad differential diagnosis of both inflammatory and tumor diseases.
Methods: 20-year-old male who visited the emergency room due to a one week decreased visual acuity on the right eye (RE). No relevant medical history. Best corrected visual acuity (BCVA) of counting fingers OD 20/20. Anterior biomicroscopy: No iridian nodules. Funduscopy showed an elevated parapapillary lesion with pigment, macular edema and a star. Optical coherence tomography (OCT) confirmed the hyper-reflecting elevated lesion with underlying shadow. Angiofluoresceingraph (AFG) showed a fluorescence interruption in early times with staining of lesion in late times. Additional tests are requested:Nuclear magnetic resonance and serology.

Results: In successive revisions both funduscopy and OCT showed a decreased thickness of the lesion and normalization of macular and papillary architecture despite which BCVA of 20/100.

Conclusions: Toxoplasma lesion in papilloma-macular bundle is sometimes accompanied by papillary edema and submacular exudation producing an image that can be confounded with a proliferative lesion. Clinical course and complementary tests helped us in the diagnosis which should be performed as early as possible for starting therapy.

\section{A New Epidescemetic Intrastromal Keratoprosthesis (Surgical Technique and Initial Results)}

\author{
J. Alió y Sanz, A. Abdelghany Ahmed, A. Vega-Estrada, \\ R. Fernández-Buenaga \\ Vissum Corporacion, Miguel Hernandez University, Alicante, \\ Spain
}

Purpose: To demonstrate the surgical procedure of a new keratoprosthesis (KeraKlear) and report the preliminary outcome.

Methods: The KeraKlear (KeraMed Inc. California, U.S.A.) is a new 'epidescemetic, non-perforating' keratoprosthesis device made of biocompatible polymer. The procedure starts with femtosecond laser to create 2 corneal dissections, the $1^{\text {st }} ; 8 \mathrm{~mm}$ in diameter at $470 \mu \mathrm{m}$ and the $2^{\text {nd }} ; 3.5 \mathrm{~mm}$ at $320 \mu \mathrm{m}$ of depth with same energy parameters ' $3 \mu \mathrm{J}$, raster method with spot separation $4 \mu \mathrm{m}$ '. The central $3.5 \mathrm{~mm}$ disc is removed and the device is implanted into the cornea and fixed with sutures. A pilot study on 10 eyes, with poor prognosis for keratoplasty, was done for initial evaluation.

Results: The device was well centered (OCT evaluated) with no infection or inflammation in 6 eyes with visual improvement in 4 of them. Retro-prosthetic inflammatory membrane developed in 2 eyes and corneal melting in 1 eye that required penetrating keratoplasty and keratoprosthesis re-implantation. One eye developed both corneal melting and retro-prosthetic inflammatory membrane ended by keratoprosthesis extrusion and total leucoma.

Conclusion: KeraKlear is a new non-perforating keratoprosthesis, easy implantable and reducing the risk of infection and necrosis. Larger, long follow-up studies are required for further evaluation. 